\title{
Interaction between light and highly confined hypersound in a silicon photonic nanowire
}

\author{
Raphaël Van Laer, ${ }^{*}$ Bart Kuyken, Dries Van Thourhout and Roel Baets \\ Photonics Research Group, Ghent University-imec, Belgium \\ Center for Nano- and Biophotonics, Ghent University, Belgium
}

In the past decade, there has been a surge in research at the boundary between photonics and phononics. Most efforts centered on coupling light to motion in a high-quality optical cavity, typically geared towards manipulating the quantum state of a mechanical oscillator. It was recently predicted that the strength of the light-sound interaction would increase drastically in nanoscale silicon photonic wires. Here we demonstrate, for the first time, such a giant overlap between near-infrared light and gigahertz sound co-localized in a small-core silicon wire. The wire is supported by a tiny pillar to block the path for external phonon leakage, trapping $10 \mathrm{GHz}$ phonons in an area below $0.1 \mu^{2}$. Since our geometry can also be studied in microrings, it paves the way for complete fusion between the fields of cavity optomechanics and Brillouin scattering. The result bodes well for the realization of optically-driven lasers/sasers, isolators and comb generators on a densely integrated silicon chip.

The diffraction of light by sound was first studied by Léon Brillouin in the early 1920s. Therefore, such inelastic scattering has long been called Brillouin scattering ${ }^{1}$. The effect is known as stimulated Brillouin scattering (SBS) when the sound is generated by a strong intensity-modulated light field. This sets the stage for a feedback loop: First, the beat note between two optical waves (called the pump and the Stokes seed) generates sound. Next, this sound creates a travelling index grating that scatters light. On the quantum level, the process annihilates pump photons while creating acoustic phonons and Doppler red-shifted Stokes photons.

In a seminal experimental study ${ }^{2}$, Brillouin scattering was viewed as a source of intense coherent sound. Later, the effect became better known as a noise source in quantum optics ${ }^{3}$ and for applications such as phononic band mapping ${ }^{4,5}$, slow and stored light ${ }^{6,7}$, spectrally pure lasing $^{8-10}$ and microwave signal processing ${ }^{11,12}$.

Traditionally ${ }^{1-4,6-19}$, the photon-phonon interaction was mediated by the material nonlinearity. Electrostriction drove the phonon creation and phonon-induced permittivity changes scattered photons. This conventional image of SBS as a bulk effect, without reference to geometry, breaks down in nanoscale waveguides. In such waveguides, boundary effects can no longer be neglected ${ }^{20}$. Thus, both electrostrictive forces and radiation pressure create acoustic waves: the former generate density variations and the latter transfers momentum to the waveguide boundaries. Equivalently, the new theory ${ }^{20}$ takes into account not only bulk permittivity changes but also the shifting material boundaries. The impressive progress in engineering radiation pressure in micro- and nanoscale systems ${ }^{21-28}$ recently inspired the prediction of enormously enhanced $\mathrm{SBS}^{20,29-31}$ in silicon nanowires. The strong optical confinement offered by these wires boosts both bulk and boundary forces. However, destructive interference between the two contributions may still completely cancel the photon-phonon coupling. The

* raphael.vanlaer@intec.ugent.be giant light-sound overlap arises particularly when both types of optical forces align with the acoustic field ${ }^{20,30}$.

Unfortunately, typical silicon-on-insulator (SOI) wires provide only weak acoustic confinement because there is little elastic mismatch between the silicon core and the silicon dioxide substrate. The large SBS strength was thus thought to be accessible only in silicon waveguides that are fully suspended in air ${ }^{20,29-32}$. This requirement severely compromises the ability to make centimeterscale interaction lengths, which are paramount to reduce the required pump power. Hence, Brillouin scattering remained elusive in silicon photonic nanowires.

\section{Results}

Here, we take the middle ground between these conflicting demands. By partially releasing a silicon wire from its substrate, we drastically improve acoustic confinement (fig.1a-c). There is still some leakage through the pillar, but it is sufficiently limited to tap the large overlap between the optical forces and the hypersonic mode (fig.1d). Moreover, this way it is straightforward to increase the interaction length. Building on this compromise, we demonstrate an order-of-magnitude performance leap in the light-sound coupling strength.

The observed mechanical mode strongly interacts with the fundamental quasi-TE optical mode (fig.1e). The main contribution to the coupling stems from the good overlap between the horizontal optical forces and displacement profile. In particular, the bulk electrostrictive forces $\mathbf{f}_{\mathrm{es}}$ and the boundary radiation pressure $\mathbf{f}_{\mathrm{rp}}$ both point in the same direction as the acoustic field $\mathbf{u}$ (fig.1d). Therefore they interfere constructively, leading to a total overlap $\langle\mathbf{f}, \mathbf{u}\rangle=\left\langle\mathbf{f}_{\mathrm{es}}, \mathbf{u}\right\rangle+\left\langle\mathbf{f}_{\mathrm{rp}}, \mathbf{u}\right\rangle$ up to twice as large as each individual component. Since the SBS gain $G_{\mathrm{SBS}}\left(\Omega_{\mathrm{m}}\right)$ at the phonon resonance frequency $\Omega_{\mathrm{m}}$ scales as $|\langle\mathbf{f}, \mathbf{u}\rangle|^{2}$, the total scattering from pump to Stokes photons may be up to four times as efficient as by electrostriction or radiation pressure individually.

Such force interference ${ }^{20,30}$ was previously studied in hybrid silicon nitride/silicon waveguides ${ }^{32}$. In that case, the light was confined to the silicon core but the sound 

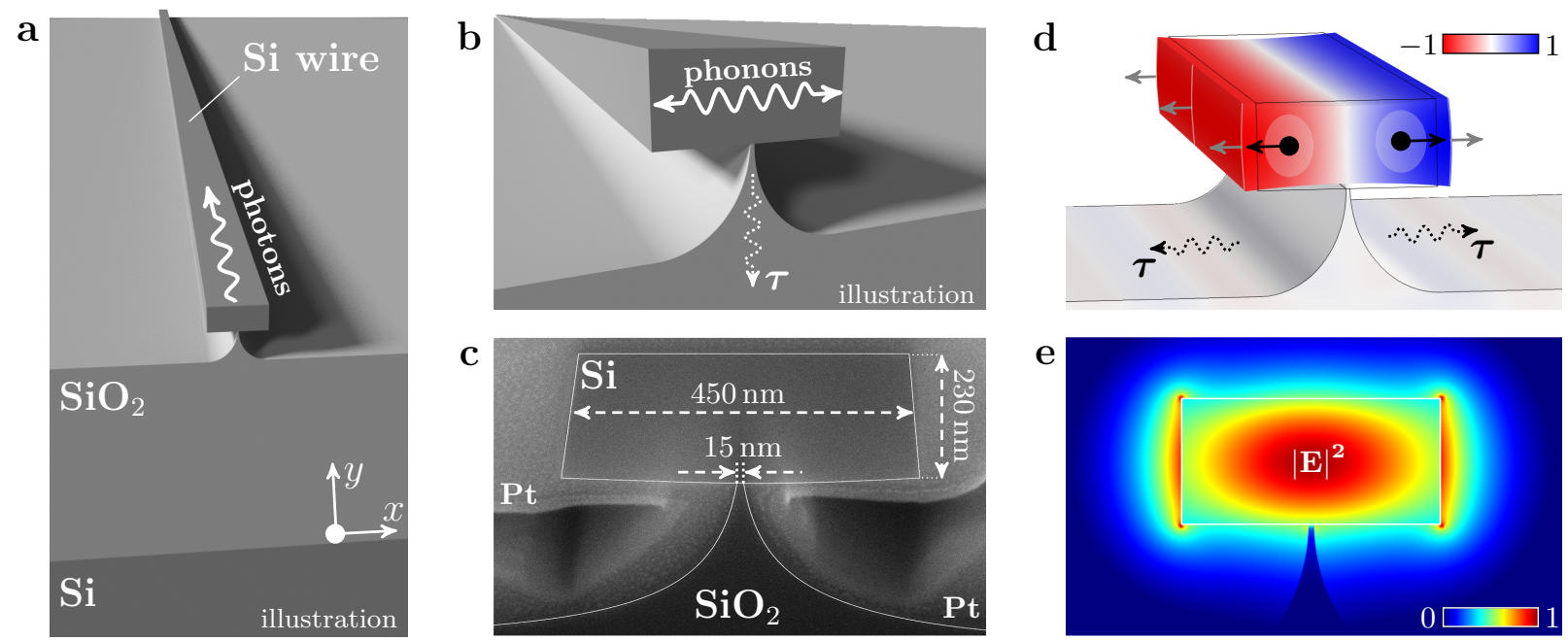

FIG. 1. A silicon wire on a pillar as an acoustic phonon cavity. a, Top view of the silicon wire. Light propagates along the wire. It confines photons owing to the high optical contrast with the silicon dioxide substrate and the air. $\mathbf{b}$, Unlike the photons, the phonons are trapped transversally. The leakage of phonons through the pillar determines their lifetime $\tau \approx 5$ ns. c, A scanning electron micrograph of the $450 \times 230 \mathrm{~nm}$ cross-section. Before ion milling, we deposit platina (Pt) on the wire for better visualization (see Methods). We fabricate pillars as narrow as $15 \mathrm{~nm}$ reliably. d, The horizontal component of the observed hypersonic mode $\mathbf{u}$ (red: - , blue: +) aligns with the bulk electrostrictive forces (black arrows) and the boundary radiation pressure (grey arrows). e, Electric field norm of the quasi-TE optical mode.

to the silicon nitride membrane. In our work, both light and sound are compressed to the same silicon core. The elastic mode (fig.1d) can be understood as the fundamental mode of a Fabry-Pérot cavity for hypersonic waves (fig.1b), formed by the silicon/air boundaries. Therefore, its frequency can be estimated as $\frac{\Omega_{\mathrm{m}}}{2 \pi}=\frac{v}{2 w}=9.4 \mathrm{GHz}$ with $v=8433 \mathrm{~m} / \mathrm{s}$ the longitudinal speed of sound in silicon and $w=450 \mathrm{~nm}$ the waveguide width.

Fabrication. To create the pillar structure, we start from an SOI wire fabricated by deep UV lithography ${ }^{33}$ through the silicon photonics platform ePIXfab (www. ePIXfab.eu). Next, we perform an additional oxide etch with hydrofluoric acid. By carefully controlling the etching speed, a narrow pillar is left underneath the wire (fig.1a-c). Through this simple fabrication method, we obtain wires up to $4 \mathrm{~cm}$ long. To retain compactness, wires longer than $3 \mathrm{~mm}$ are coiled up into a low-footprint spiral (see Supplementary Information). Despite the additional etch, the wires still exhibit optical propagation losses $\alpha$ of only $2.6 \mathrm{~dB} / \mathrm{cm}$.

Experiments. In our experiments (fig.2), we investigate straight and spiral waveguides with lengths $L$ ranging from $1.4 \mathrm{~mm}$ to $4 \mathrm{~cm}$. We couple $1550 \mathrm{~nm}$ TE-light to the waveguides through focusing grating couplers ${ }^{34}$ and perform both gain (fig.2a-b) and cross-phase modulation (fig.2c-d) experiments. The resonances (fig.2a and c) observed in these experiments allow for a characterization of the photon-phonon coupling in two independent ways.

Gain. First, we monitor the power in a Stokes seed as a function of frequency spacing $\frac{\Omega}{2 \pi}$ with a strong co-propagating pump wave (fig.2a-b). We observe a Lorentzian gain profile at $\frac{\Omega_{\mathrm{m}}}{2 \pi}=9.2 \mathrm{GHz}$, as expected in the low-cascading regime (see SI). Similarly, we ob- serve an identical depletion profile on an anti-Stokes seed (fig.2a). The Stokes seed experiences amplification as long as the pump remains undepleted. Exactly on resonance, the on/off gain is given by $2 \gamma_{\mathrm{SBS}} P_{\mathrm{p}} L_{\mathrm{eff}}-$ with $2 \gamma_{\mathrm{SBS}}=G_{\mathrm{SBS}}\left(\Omega_{\mathrm{m}}\right)$ the Brillouin gain coefficient, $P_{\mathrm{p}}$ the input pump power and $L_{\text {eff }}=\frac{1-\exp (-\alpha L)}{\alpha}$ the effective interaction length. The effective length has an upper limit of $\frac{1}{\alpha}=1.7 \mathrm{~cm}$ in our wires. To extract the Brillouin parameter $\gamma_{\mathrm{SBS}}$, we sweep the pump power in a $2.7 \mathrm{~mm}$ long wire (fig.3a). Above $25 \mathrm{~mW}$ on-chip power, nonlinear absorption saturates the on/off gain. Then free carriers, created by two-photon absorption (TPA), result in a power-dependent optical loss $\alpha\left(P_{\mathrm{p}}\right)$. We extract $2 \gamma_{\mathrm{SBS}}=$ $3218 \mathrm{~W}^{-1} \mathrm{~m}^{-1}$ below this threshold. The Lorentzian fit yields an acoustic linewidth of $\frac{\Gamma_{\mathrm{m}}}{2 \pi}=30 \mathrm{MHz}$ and thus a quality factor of $Q_{\mathrm{m}}=\frac{\Omega_{\mathrm{m}}}{\Gamma_{\mathrm{m}}}=306$ and a phonon lifetime of $\tau=\frac{1}{\Gamma_{\mathrm{m}}}=5.3 \mathrm{~ns}$ in the same short wire. The largest on/off gain of $0.6 \mathrm{~dB}$ below the TPA-threshold falls narrowly short of the linear loss $\alpha L=0.7 \mathrm{~dB}$ (fig.3a). Thus the wire is close to net optical amplification, which is necessary for Brillouin lasing. The on/off gain reaches $175 \%$ in the longest $4 \mathrm{~cm}$-wires (fig.2a), improving by a factor 19 on previous results in silicon ${ }^{32}$.

Forward vs. backward. Similarly, we observe backward SBS (see SI): for counter-propagating pump and Stokes waves that generate elastic waves with a large wavevector $K \approx 2 k_{0}$ and $k_{0}$ the pump wavevector. However, we achieve the giant light-sound overlap only for forward SBS: for co-propagating pump and Stokes waves that generate low group velocity acoustic phonons with small wavevector $K \approx \frac{\Omega}{v_{\mathrm{g}}}$ and $v_{\mathrm{g}}$ the optical group velocity. Therefore, we focus on forward SBS here. 


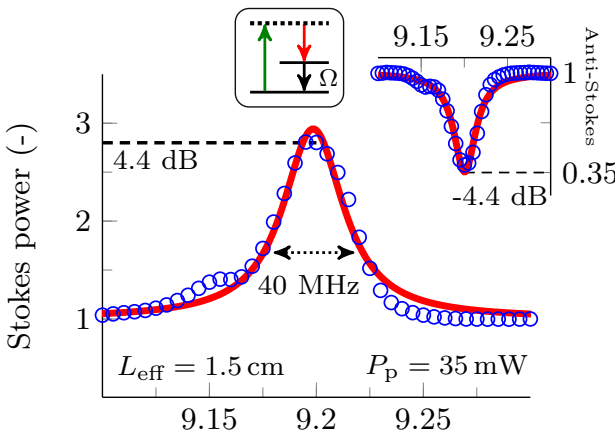

$\mathbf{a}$
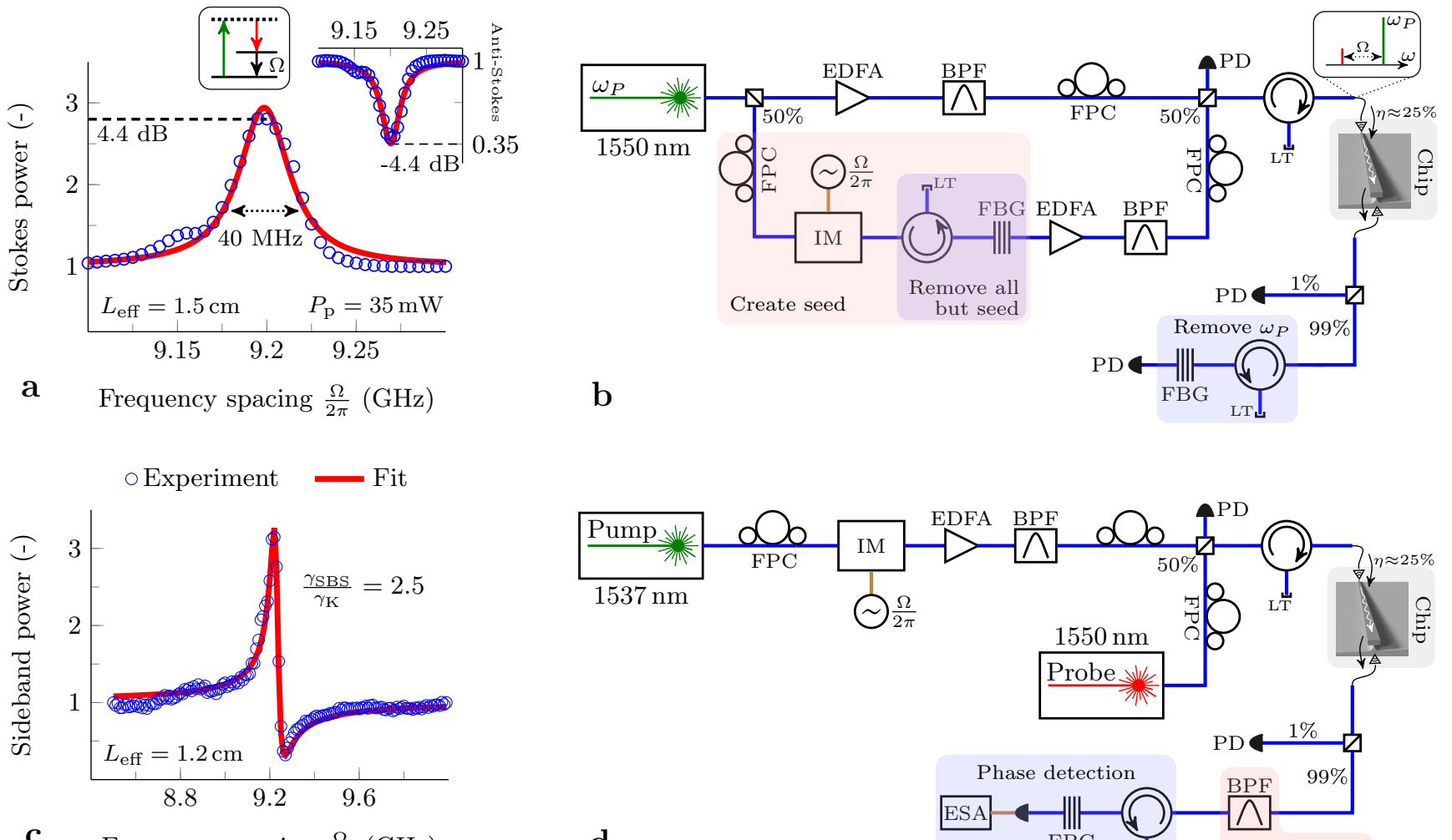

c Frequency spacing $\frac{\Omega}{2 \pi}(\mathrm{GHz})$

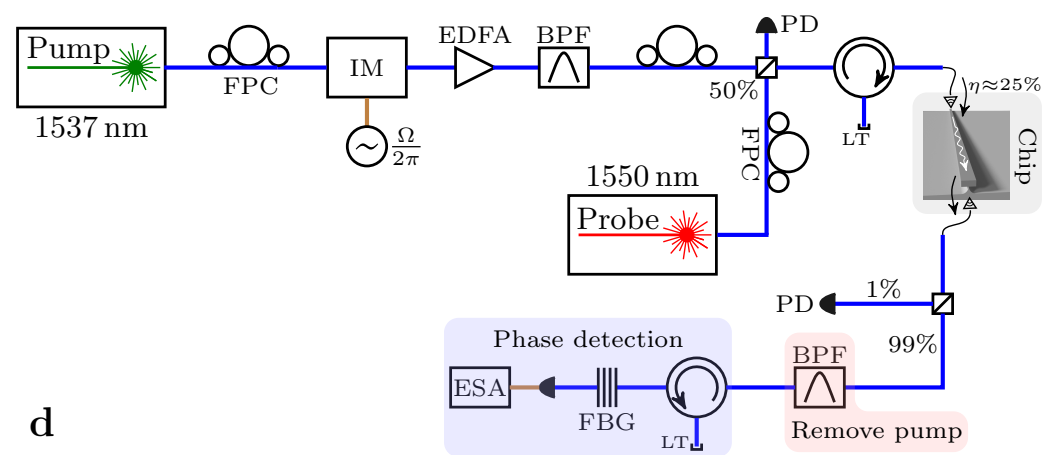

FIG. 2. Experimental characterization of the photon-phonon coupling. a, A typical Lorentzian gain profile on a Stokes seed and (inset) a depletion profile on an anti-Stokes seed. In both cases, the interaction generates acoustic phonons and red-shifted photons (energy diagram). We observe such resonances in wires as short as $2.7 \mathrm{~mm}$ (see SI) and obtain the highest on/off gain of $175 \%(4.4 \mathrm{~dB})$ in the $4 \mathrm{~cm}$ spiral $\left(L_{\mathrm{eff}}=1.5 \mathrm{~cm}\right)$ with $35 \mathrm{~mW}$ on-chip pump power. There is a remnant of a second resonance at $9.15 \mathrm{GHz}$ (see SI). We fit the normalized Stokes power to the exponential of a Lorentzian function (see SI formula (6)). b, The fiber-based set-up used to study forward SBS. A tunable laser is amplified in the upper arm by an erbium-doped fiber amplifier (EDFA) to serve as a pump. In the lower arm, the laser light is intensity modulated (IM) to generate a blue- and red-shifted sideband. Next, a fiber Bragg grating (FBG) rejects all but the Stokes seed (see Methods). The pump and Stokes seed are coupled to the chip through curved grating couplers. Finally, the power in the pump and Stokes wave is monitored separately. Light traps (LTs) prevent backscattered light from entering the circuit. With minor modifications, this set-up can be reconfigured to observe the loss on an anti-Stokes seed or the backward SBS. The latter is weak in our wires (see SI), so we focus on the forward SBS. c, A typical Fano signature obtained from the XPM-experiment, which we use to calibrate the Brillouin with respect to the Kerr nonlinearity $\left(\gamma_{\mathrm{SBS}} / \gamma_{\mathrm{K}}=2.5\right)$. d, A pump is intensity modulated, amplified, combined with a probe wave and sent to the chip. The pump is removed at the output by a band-pass filter (BPF). The phase modulation on the probe wave is transducted to intensity modulation by filtering out the red-shifted sideband. Finally, we use an electrical spectrum analyzer (ESA) to observe the beat between the probe and the imprinted blue sideband.

Cross-phase modulation. Second, we measure the strength of the cross-phase modulation (XPM) imprinted on a weak probe by a strong intensity-modulated pump (fig.2c-d). The experiment yields a distinct asymmetric Fano signature at $\frac{\Omega_{\mathrm{m}}}{2 \pi}=9.2 \mathrm{GHz}$ caused by interference between the resonant Brillouin and the non-resonant Kerr response (see SI). The lineshape follows $\left|\frac{\gamma_{\mathrm{XPM}}(\Omega)}{2 \gamma_{\mathrm{K}}}\right|^{2}$, with $\gamma_{\mathrm{K}}$ the Kerr parameter and

$$
\begin{gathered}
\gamma_{\mathrm{XPM}}(\Omega)=2 \gamma_{\mathrm{K}}+\gamma_{\mathrm{SBS}} \mathcal{L}(\Omega) \\
\mathcal{L}(\Omega)=\frac{1}{-2 \Delta_{r}+i} \quad \Delta_{r}=\frac{\Omega-\Omega_{\mathrm{m}}}{\Gamma_{\mathrm{m}}}
\end{gathered}
$$

We deduce the ratio $\gamma_{\mathrm{SBS}} / \gamma_{\mathrm{K}}=2.5$ and $Q_{\mathrm{m}}=249$ from the fit. The Kerr parameter $\gamma_{K}$ of similar silicon wires has been studied extensively, with values reported at $\gamma_{\mathrm{K}}=566 \mathrm{~W}^{-1} \mathrm{~m}^{-1}$ for our cross-section ${ }^{35}$. Because of the pillar etch, the light is more confined to the highindex silicon core. We simulate that this yields a slight increase of the Kerr effect by $8 \%$ to $\gamma_{\mathrm{K}}=611 \mathrm{~W}^{-1} \mathrm{~m}^{-1}$. Thus we have $2 \gamma_{\mathrm{SBS}}=3055 \mathrm{~W}^{-1} \mathrm{~m}^{-1}$, within $5 \%$ of the value obtained from the gain experiments. This nonlinearity is at least a factor $10^{3}$ stronger than in photonic crystal and highly nonlinear fibres ${ }^{15,36}$. Further, the resonance frequency, quality factor and interaction strength are in good agreement with the models.

Resonance frequency. To study the frequency, we perform the XPM-experiment for waveguide widths from $350 \mathrm{~nm}$ to $500 \mathrm{~nm}$ (fig.3b). Both a simple Fabry-Pérot $\left(\frac{\Omega_{\mathrm{m}}}{2 \pi}=\frac{v}{2 w}\right)$ and a sophisticated finite-element model match the observed resonances. The finite-element model 


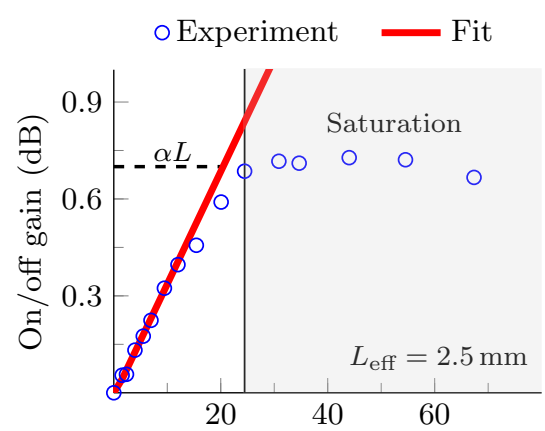

a $\quad$ On-chip pump power $P_{\mathrm{p}}(\mathrm{mW})$

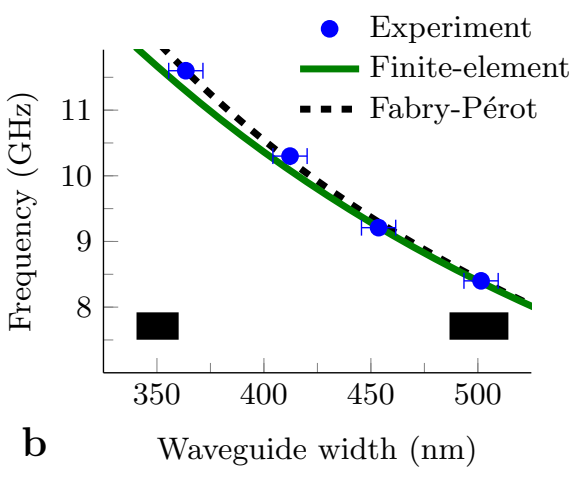

FIG. 3. Analysis of the Brillouin gain and phononic resonance frequency. a, Scaling of the on/off Brillouin gain with input pump power. Above a power threshold of $25 \mathrm{~mW}$, the on/off gain saturates because of nonlinear absorption. We perform a fit to obtain the Brillouin nonlinearity below that threshold. $\mathbf{b}$, The phononic resonance frequency for different waveguide widths. Both a simple Fabry-Pérot and a rigorous finite-element model agree with the data.

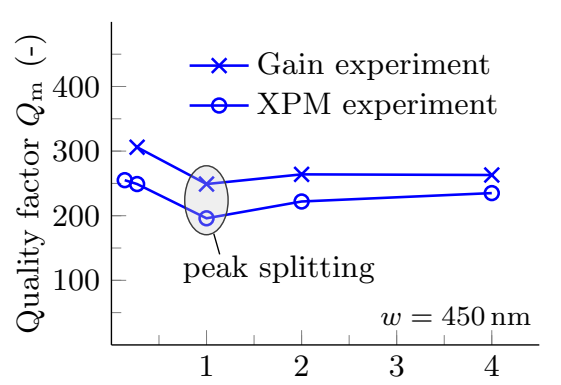

a

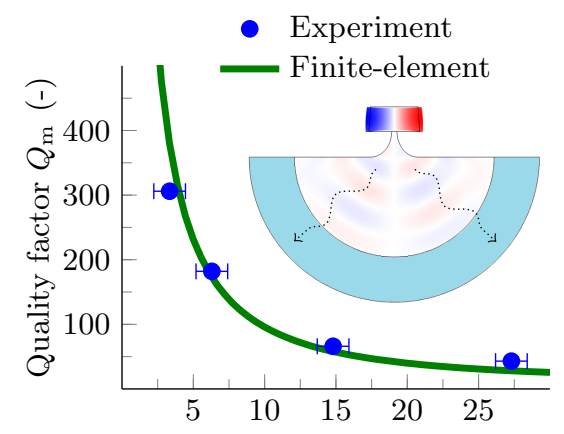

b Pillar relative to wire width $(\%)$

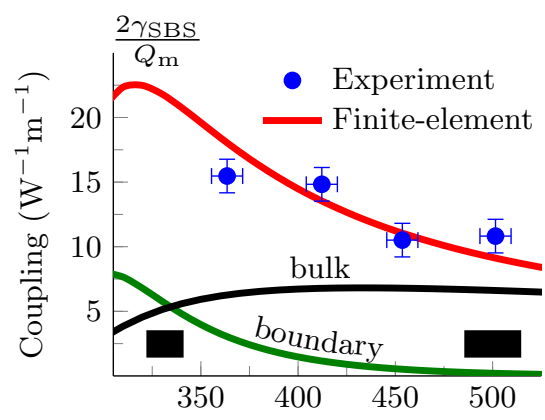

C Waveguide width $(\mathrm{nm})$

FIG. 4. Study of the mechanical quality factor and the intrinsic photon-phonon overlap. a, The quality factor stays above 250 even in $4 \mathrm{~cm}$-long spirals, showing no length-dependent line broadening. Besides, there is peak splitting in the $1 \mathrm{~cm}$ spiral (see SI). Neither the longer spirals nor the straight wires exhibit such splitting. $\mathbf{b}$, A finite-element model of phonon leakage through the pillar accurately predicts the observed quality factors $(L=2.7 \mathrm{~mm})$. c, The non-resonant nonlinearity $\frac{2 \gamma_{\mathrm{SBS}}}{Q_{m}}$ is a measure of the intrinsic photon-phonon coupling. The electrostriction (black) and radiation pressure (green) interfere constructively, bringing about the total overlap (red). As the width increases, the boundary contribution vanishes rapidly.

takes into account the exact geometry of the wires as obtained from a scanning electron micrograph (fig.1c). This includes the waveguide height, pillar size, sidewall angle and the $\langle 110\rangle$ crystal orientation of our wires (see Methods). We find that the waveguide width alone pins down the resonance frequency, with other geometrical parameters inducing minor shifts. For a $450 \mathrm{~nm}$-wide waveguide, the frequency sensitivity to width changes is $19.2 \mathrm{MHz} / \mathrm{nm}$ (fig.3b). In contrast, the calculated sensitivity to height changes is only $2.3 \mathrm{MHz} / \mathrm{nm}$. This supports the intuitive Fabry-Pérot view, in which the height does not appear at all.

The large sensitivity to waveguide width implies that a $2 \mathrm{~nm}$ width fluctuation shifts the resonance by more than a linewidth. Therefore inhomogeneous broadening may affect both the lineshape and -width, similar to Doppler-broadening in gain media. Surprisingly, we achieve acoustic quality factors above 250 even in the longest $4 \mathrm{~cm}$-wires (fig.4a). This suggests that there is, if at all, only limited length-dependent line broadening (see SI). Besides, this large sensitivity can be exploited to tailor the resonance frequency.

Quality factor. By sweeping the pillar size in a short $450 \mathrm{~nm}$-wide waveguide, we establish leakage through the pillar as the dominant phononic loss mechanism (fig.4b). The pillar acts as a channel for elastic waves that propagate down into the substrate. We rigorously model this mechanism by adding an artificial absorbing layer at the boundary of the simulation domain (see Methods). As predicted by such a model, the observed quality factors diminish rapidly with increasing pillar size. The pillar should be seen as a moving acoustic membrane ${ }^{37}$, not as a fixed point. Therefore, it affects neither the acoustic field profile nor its associated stiffness $k_{\text {eff }}$ considerably. We regard a short phonon lifetime as the prime reason that SBS was not observed so far in typical SOI nanowires.

Interaction strength. Finally, we show that the photon-phonon coupling is a constructive combination of bulk (electrostriction) and boundary (radiation pressure) effects (fig.4c). The resonant Brillouin gain coefficient 
is given by $G_{\mathrm{SBS}}\left(\Omega_{\mathrm{m}}\right)=2 \gamma_{\mathrm{SBS}}=\omega_{0} Q_{\mathrm{m}}|\langle\mathbf{f}, \mathbf{u}\rangle|^{2} /\left(2 k_{\mathrm{eff}}\right)$ (see SI), so the non-resonant part $\frac{2 \gamma_{\mathrm{SBS}}}{Q_{\mathrm{m}}}$ is a direct measure of the photon-phonon overlap ${ }^{20,30}$. In our finiteelement simulations of $\langle\mathbf{f}, \mathbf{u}\rangle$ and $k_{\text {eff }}$, we take into account the mechanical anisotropy of silicon but not the pillar. We also approximate the cross-section as rectangular, neglecting the small sidewall angle. Still, the simulations match the experimentally determined coupling strength. Neither electrostriction nor radiation pressure separately explain the experimental values of $\frac{2 \gamma_{\mathrm{SBS}}}{Q_{\mathrm{m}}} \approx 12 \mathrm{~W}^{-1} \mathrm{~m}^{-1}$. These values are a factor 10 larger than in on-chip chalcogenide ${ }^{19}$ and silicon nitride/silicon waveguides $^{32}$, showing the benefit of compressing light and sound to the same nanoscale core.

\section{Discussion}

Our observations provide robust evidence for incredibly strong photon-phonon coupling in silicon nanowires. The simulations of the interaction strength match the experiments, indicating that the new nanoscale SBS theory ${ }^{20}$ is on the right track. Moreover, simple finite-element models accurately capture both the phononic resonance frequency and lifetime.

Building on the good light-sound overlap, some typical SBS applications are now squarely in reach. For lasing ${ }^{38}$, the gain must exceed the loss over an optical cavity roundtrip. Currently, we achieve $0.6 \mathrm{~dB}$ on/off gain in a wire with $0.7 \mathrm{~dB}$ propagation loss (fig. $3 \mathrm{a}$ ). We note that lasing - as opposed to sasing - also requires a resonator that is less optically than acoustically damped ${ }^{10,38-40}$. Another example is the microwave filter ${ }^{12}$, since such filters can be driven by even sub- $1 \mathrm{~dB}$ gain ${ }^{41}$.

For other devices, such as isolators ${ }^{16,42}$ and slow light ${ }^{7}$, the performance in terms of optical losses and SBS strength must be improved more substantially. Optical losses below $1 \mathrm{~dB} / \mathrm{cm}$ have already been demonstrated in comparable silicon wires; by moving from a $200 \mathrm{~mm}$ to a $300 \mathrm{~mm}$-wafer CMOS pilot line with more advanced lithography tools ${ }^{43}$. Significant net gain should be accessible in such low-loss wires, in which effective interaction lengths up to $L_{\mathrm{eff}}=5 \mathrm{~cm}$ may be obtained.

Further, free-carrier absorption saturates the SBS gain above a pump power of $25 \mathrm{~mW}$ (fig.3a). Therefore, the SBS gain has to be improved by other means: either the phonon lifetime $\tau$ or the photon-phonon overlap $\langle\mathbf{f}, \mathbf{u}\rangle$ should be enhanced. Currently limited by the phonon leakage, the lifetime could be increased - at the cost of smaller bandwidth - by exciting asymmetric elastic modes ${ }^{44}$. It has been predicted that such modes can be generated efficiently by cross-coupling between the quasi-TE and quasi-TM optical mode ${ }^{30}$. Alternatively, the overlap $\langle\mathbf{f}, \mathbf{u}\rangle$ may be increased by trapping light in a narrow horizontal air-slot between two specially designed silicon wires ${ }^{31}$. Such ideas may further boost the Brillouin nonlinearity to a level sufficient for milliwattthreshold lasing ${ }^{38,39}$, frequency comb generation ${ }^{15,45}$ and fully non-resonant optomechanics ${ }^{46}$.
In fact, each application comes with a specific figure of merit. For comb generation with a dual-pump ${ }^{15}$, the forward SBS gain is critical. Then it is equivalent to increase the lifetime $\tau$ or the overlap $\langle\mathbf{f}, \mathbf{u}\rangle$. In other cases, such as for slow light ${ }^{7}$, the bandwidth is equally important. Further, it is often desirable to have the acoustic resonance in the gigahertz range ${ }^{12}$ - which implicitly sets the stiffness $k_{\text {eff }}$ given a certain mass. Even so, a large light-sound overlap $\langle\mathbf{f}, \mathbf{u}\rangle$ - clearly manifested in this work - is greatly beneficial in all cases.

In conclusion, we demonstrated efficient interaction between near-infrared light and gigahertz sound trapped in a small-core silicon photonic wire. The structure exhibits an extraordinary light-sound overlap, at the same time allowing for a centimeter-scale Brillouin-active interaction length. The combination of both opens the door to practical Brillouin devices integrated on a CMOScompatible silicon chip.

\section{Methods}

Device fabrication and characterization. The SOI wires are fabricated by $193 \mathrm{~nm}$ deep UV lithography on a $200 \mathrm{~mm}$ wafer CMOS pilot line at imec. We underetch the wires with $2 \%$ diluted hydrofluoric acid at an etching rate of $10 \mathrm{~nm} / \mathrm{min}$. We deposit platina $(\mathrm{Pt})$ on the wire and then mill the cross-section (fig.1c) by a focused ion beam. The platina deposition ensures a straight cross-section and prevents charging effects when the cross-section is viewed by a scanning electron beam. The straight wires have lengths 1.4 and $2.7 \mathrm{~mm}$, while the spirals are 1,2 and $4 \mathrm{~cm}$ long (see SI). The 1,2 and $4 \mathrm{~cm}$ spirals have a footprint of $275 \mu \mathrm{m} \times 100 \mu \mathrm{m}$, $775 \mu \mathrm{m} \times 90 \mu \mathrm{m}$ and $1570 \mu \mathrm{m} \times 90 \mu \mathrm{m}$ respectively. Adjacent wires are spaced by $1.55 \mu \mathrm{m}$ inside the spiral. We find the propagation loss $\alpha$ of $2.6 \mathrm{~dB} / \mathrm{cm}$ and the coupling loss of $6 \mathrm{~dB}$ per grating coupler by the cut-back method.

Experimental set-up. We use the following abbreviations (fig.2): erbium-doped fibre amplifier (EDFA), bandpass filter (BPF), fibre polarization controller (FPC), intensity modulator (IM), electrical spectrum analyser (ESA), light trap (LT), fibre Bragg grating (FBG) and photodetector (PD). The FBGs were a crucial part of our set-up. Produced by TeraXion Inc., these filters were custom-designed to have a flat response within the passband and drop to $-30 \mathrm{~dB}$ within $2.5 \mathrm{GHz}$. We use the steep flanks to filter out either the redor blue-shifted sidebands. Their bandwidth is $60 \mathrm{GHz}$. In addition, we employ a pair of perfectly aligned FBGs for the gain experiment (fig. $2 \mathrm{~b}$ ).

Finite-element modelling. We obtain the photonic and phononic modes from the finite-element solver COMSOL. They were exported to MATLAB to calculate the photonphonon coupling ${ }^{20,30}$. Since our wires are aligned along a $\langle 110\rangle$ axis, we rotated both the elasticity $\left(c_{11}, c_{12}, c_{44}\right)=$ $(166,64,79) \mathrm{GPa}$ and the photoelasticity $\left(p_{11}, p_{12}, p_{44}\right)=$ $(-0.09,0.017,-0.051)$ matrix by $\pi / 4$. To simulate the clamping loss, we add an artificial silica matching layer with Young's modulus $\frac{i}{\zeta} E$ and density $-i \zeta \rho$. The layer absorbs incoming elastic waves without reflection. In a frequency-domain simulation, we then find the quality factor from $Q_{\mathrm{m}}=\frac{\Re \Omega_{\mathrm{m}}}{2 \Im \Omega_{\mathrm{m}}}$. We optimize $\zeta$ for minimal $Q_{\mathrm{m}}$. A typical value is $\zeta \stackrel{2 \Im \Omega_{\mathrm{m}}}{=}$ for a $420 \mathrm{~nm}$-thick matching layer. 
[1] Boyd, R. Nonlinear optics (Elsevier, London, 2008), 3 edn.

[2] Chiao, R., Townes, C. \& Stoicheff, B. Stimulated Brillouin scattering and coherent generation of intense hypersonic waves. Phys. Rev. Lett. (1964).

[3] Shelby, R., Levenson, M. \& Bayer, P. Resolved forward Brillouin scattering in optical fibers. Phys. Rev. Lett. 54, 939-942 (1985).

[4] Gorishnyy, T., Ullal, C., Maldovan, M., Fytas, G. \& Thomas, E. Hypersonic phononic crystals. Phys. Rev. Lett. 94, 115501 (2005).

[5] Maldovan, M. Sound and heat revolutions in phononics. Nature 503, 209-217 (2013).

[6] Zhu, Z., Gauthier, D. \& Boyd, R. Stored light in an optical fiber via stimulated Brillouin scattering. Science 318, 1748-50 (2007).

[7] Thévenaz, L. Slow and fast light in optical fibres. Nature Photon. 2 (2008).

[8] Grudinin, I., Matsko, A. \& Maleki, L. Brillouin lasing with a $\mathrm{CaF}_{2}$ whispering gallery mode resonator. Phys. Rev. Lett. 102, 043902 (2009).

[9] Tomes, M. \& Carmon, T. Photonic microelectromechanical systems vibrating at X-band (11-GHz) rates. Phys. Rev. Lett. 102, 113601 (2009).

[10] Lee, H. et al. Chemically etched ultrahigh-Q wedgeresonator on a silicon chip. Nature Photon. 6, 369-373 (2012).

[11] Li, J., Lee, H. \& Vahala, K. Microwave synthesizer using an on-chip Brillouin oscillator. Nat. Commun. 4, 1-7 (2013).

[12] Pant, R. et al. On-chip stimulated Brillouin scattering for microwave signal processing and generation. Laser Photon. Rev. (2014).

[13] Dainese, P. et al. Stimulated Brillouin scattering from multi-GHz-guided acoustic phonons in nanostructured photonic crystal fibres. Nature Phys. 2, 388-392 (2006).

[14] Kobyakov, A., Sauer, M. \& Chowdhury, D. Stimulated Brillouin scattering in optical fibers. Adv. Opt. Photon. 2, 1 (2009).

[15] Kang, M., Nazarkin, A., Brenn, A. \& Russell, P. Tightly trapped acoustic phonons in photonic crystal fibres as highly nonlinear artificial Raman oscillators. Nature Phys. 5, 276-280 (2009).

[16] Kang, M., Butsch, A. \& Russell, P. Reconfigurable lightdriven opto-acoustic isolators in photonic crystal fibre. Nature Photon. 5, 549-553 (2011).

[17] Bahl, G., Zehnpfennig, J., Tomes, M. \& Carmon, T. Stimulated optomechanical excitation of surface acoustic waves in a microdevice. Nat. Commun. 2, 403 (2011).

[18] Bahl, G., Tomes, M., Marquardt, F. \& Carmon, T. Observation of spontaneous Brillouin cooling. Nature Phys. 8, 203-207 (2012).

[19] Eggleton, B., Poulton, C. \& Pant, R. Inducing and harnessing stimulated Brillouin scattering in photonic integrated circuits. Adv. Opt. Photon. 536-587 (2013).

[20] Rakich, P., Reinke, C., Camacho, R., Davids, P. \& Wang, Z. Giant enhancement of stimulated Brillouin scattering in the subwavelength limit. Phys. Rev. X 2, 1-15 (2012).

[21] Kippenberg, T. \& Vahala, K. Cavity optomechanics: back-action at the mesoscale. Science 321, 1172-6 (2008).
[22] Li, M. et al. Harnessing optical forces in integrated photonic circuits. Nature 456, 480-4 (2008).

[23] Li, M., Pernice, W. \& Tang, H. Tunable bipolar optical interactions between guided lightwaves. Nature Photon. 3, 464-468 (2009).

[24] Roels, J. et al. Tunable optical forces between nanophotonic waveguides. Nature Nanotech. 4, 510-3 (2009).

[25] Wiederhecker, G., Chen, L., Gondarenko, A. \& Lipson, M. Controlling photonic structures using optical forces. Nature 462, 633-6 (2009).

[26] Van Thourhout, D. \& Roels, J. Optomechanical device actuation through the optical gradient force. Nature Photon. 4, 211-217 (2010).

[27] Grudinin, I., Lee, H., Painter, O. \& Vahala, K. Phonon laser action in a tunable two-level system. Phys. Rev. Lett. 104, 083901 (2010).

[28] Chan, J. et al. Laser cooling of a nanomechanical oscillator into its quantum ground state. Nature 478, 89-92 (2011).

[29] Okawachi, Y. \& Gaeta, A. Nonlinear photonics: compressing light and sound. Nature Photon. 6, 274-276 (2012)

[30] Qiu, W. et al. Stimulated Brillouin scattering in nanoscale silicon step-index waveguides: a general framework of selection rules and calculating SBS gain. Opt. Express 21, 31402 (2013).

[31] Van Laer, R., Kuyken, B., Van Thourhout, D. \& Baets, R. Analysis of enhanced stimulated Brillouin scattering in silicon slot waveguides. Opt. Lett. 39, 1242-5 (2014).

[32] Shin, H. et al. Tailorable stimulated Brillouin scattering in nanoscale silicon waveguides. Nat. Commun. 4, 1944 (2013).

[33] Bogaerts, W. et al. Nanophotonic waveguides in siliconon-insulator fabricated with CMOS technology. J. Lightwave Technol. 23, 401-412 (2005).

[34] Van Laere, F. et al. Compact focusing grating couplers for silicon-on-insulator integrated circuits. IEEE Photon. Technol. Lett. 19, 1919-1921 (2007).

[35] Osgood, R. et al. Engineering nonlinearities in nanoscale optical systems: physics and applications in dispersionengineered silicon nanophotonic wires. Adv. Opt. Photon. 1, 162 (2009).

[36] Wang, J., Zhu, Y., Zhang, R. \& Gauthier, D. FSBS resonances observed in a standard highly nonlinear fiber. Opt. Express 19, 5339-49 (2011).

[37] Anetsberger, G., Rivière, R., Schliesser, A., Arcizet, O. \& Kippenberg, T. Ultralow-dissipation optomechanical resonators on a chip. Nature Photon. 2, 627-633 (2008).

[38] Kabakova, I. Narrow linewidth Brillouin laser based on chalcogenide photonic chip. Opt. Lett. 38, 3208-11 (2013).

[39] Li, J., Lee, H. \& Vahala, K. Low-noise Brillouin laser on a chip at $1064 \mathrm{~nm}$. Opt. Lett. 39, 287-90 (2014).

[40] Nunnenkamp, A., Sudhir, V., Feofanov, A., Roulet, A. \& Kippenberg, T. Quantum-limited amplification and parametric instability in the reversed dissipation regime of cavity optomechanics. Phys. Rev. Lett. 113, 023604 (2014).

[41] Marpaung, D., Morrison, B., Pant, R. \& Eggleton, B. Frequency agile microwave photonic notch filter with anomalously high stopband rejection. Opt. Lett. 38, 
4300-3 (2013).

[42] Huang, X. \& Fan, S. Complete all-optical silica fiber isolator via stimulated Brillouin scattering. J. Lightwave Technol. 29, 2267-2275 (2011).

[43] Selvaraja, S. et al. Highly uniform and low-loss passive silicon photonics devices using a $300 \mathrm{~mm}$ CMOS platform. OFC 3-5 (2014).

[44] Wilson-Rae, I. \& Barton, R. High-Q nanomechanics via destructive interference of elastic waves. Phys. Rev. Lett. 047205, 1-4 (2011).

[45] Butsch, A., Koehler, J.,t Noskov, R. \& Russell, P. CWpumped single-pass frequency comb generation by resonant optomechanical nonlinearity in dual-nanoweb fiber. Optica 1, 158-164 (2014).

[46] Pernice, W., Li, M. \& Tang, H. A mechanical Kerr effect in deformable photonic media. Appl. Phys. Lett. 95,
123507 (2009).

Acknowledgement. R.V.L. acknowledges the Agency for Innovation by Science and Technology in Flanders (IWT) for a $\mathrm{PhD}$ grant. This work was partially funded under the FP7-ERC-InSpectra programme and the ITN-network cQOM. R.V.L. thanks T. Van Vaerenbergh for reading the manuscript and L. Van Landschoot for taking SEM-images.

Author contributions. R.V.L. performed the fabrication, experiments, analysis and wrote the paper. B.K. gave experimental and conceptual advice. D.V.T. and R.B. supervised the work. All authors discussed the results and provided feedback on the manuscript.

Additional information. Supplementary information is available in the online version of the paper. Reprints and permissions information is available online at www.nature. com/reprints. Correspondence and requests for materials should be addressed to R.V.L. 


\title{
Supplementary information for \\ "Interaction between light and highly confined hypersound in a silicon photonic nanowire"
}

\author{
Raphaël Van Laer, ${ }^{*}$ Bart Kuyken, Dries Van Thourhout and Roel Baets \\ Photonics Research Group, Ghent University-imec, Belgium \\ Center for Nano- and Biophotonics, Ghent University, Belgium
}

(Dated: January 5, 2015)

\section{CONTENTS}

I. Model of the gain experiments

II. The Brillouin gain coefficient

III. Model of the XPM experiments

IV. Measurement of the free-carrier lifetime

V. Inhomogeneous broadening

VI. Peak splitting in spirals

VII. Measurement of backward SBS

References

\section{MODEL OF THE GAIN EXPERIMENTS}

In this section we derive a model that captures the essential dynamics of forward stimulated Brillouin scattering in the presence of a background Kerr effect. Our analysis is very similar to earlier discussions of forward $\mathrm{SBS}^{\mathrm{S} 1, \mathrm{~S} 2}$ and Raman scattering ${ }^{\mathrm{S} 3}$. Specifically, we describe under which circumstances forward SBS can still be seen as a pure gain process. Thus the model includes

- cascading into higher-order Stokes and anti-Stokes waves,

- four-wave mixing contributions from both the Brillouin and the Kerr effect and

- the effect of these contributions on the SBS gain.

We assume that the electromagnetic field is composed of discrete lines, with $a_{n}(z)$ the complex amplitude of component $n$ with angular frequency $\omega_{n}=\omega_{0}+n \Omega$ at position $z$ along the guide. By definition, $\omega_{0}$ is the frequency of the pump. In the presence of weak nonlinear coupling between the waves, the evolution of the slowly-varying amplitudes is ${ }^{\mathrm{S} 3}$

$$
\frac{\mathrm{d} a_{n}}{\mathrm{~d} z}=-i \frac{\omega_{0}}{2 c n_{\mathrm{eff}} \epsilon_{0}} p_{n}^{\mathrm{NL}}
$$

\footnotetext{
* raphael.vanlaer@intec.ugent.be
}

with $p_{n}^{\mathrm{NL}}$ the complex amplitude at frequency $\omega_{n}$ of the nonlinear polarization $P^{\mathrm{NL}}(z, t)=\epsilon_{0} \chi^{\mathrm{NL}}(z, t) E(z, t)$ with $E(z, t)=\frac{1}{2} \sum_{n} a_{n}(z) \exp \left(i\left(\omega_{n} t-k_{n} z\right)\right)+$ c.c. the electric field. Here we assumed that the cascading is limited to tens of higher-order sidebands, such that $\omega_{n} \approx \omega_{0}$ and that all components experience the same effective mode index $n_{\text {eff }}$. Further, the nonlinear susceptibility is given by

$$
\chi^{\mathrm{NL}}(z, t)=2 n_{\mathrm{eff}} \Delta n_{\mathrm{eff}}(z, t)
$$

in case the index changes $\Delta n_{\mathrm{eff}}(z, t)$ are small. These index changes are composed of an instantaneous Kerr component and a delayed Brillouin component:

$$
\begin{aligned}
\Delta n_{\mathrm{eff}}(z, t) & =\Delta n_{\mathrm{eff}, \operatorname{Kerr}}(z, t)+\Delta n_{\mathrm{eff}, \text { Brillouin }}(z, t) \\
& =\frac{\bar{n}_{2}}{A_{\mathrm{eff}}} P(z, t)+\left.\frac{\partial n_{\mathrm{eff}}}{\partial q}\right|_{q_{\mathrm{avg}}} q(z, t)
\end{aligned}
$$

with $\bar{n}_{2}$ the nonlinear Kerr index averaged over the waveguide cross-section, $A_{\text {eff }}$ the effective mode area, $P(z, t)$ the total optical power and $q$ a coordinate describing the mechanical motion. There is a small shift in the average value of $q$ due to the constant component of the power $P(z, t)$. However, we can always redefine $q$ such that $q_{\text {avg }} \equiv 0$. In addition, $\frac{\partial n_{\text {eff }}}{\partial q}$ is the sensitivity of the effective index with respect to motion. This factor contains contributions from both the moving boundary (radiation pressure) and the bulk (electrostriction). It should be calculated at fixed optical frequency, since this frequency is externally applied. We characterize the mechanical mode as a harmonic oscillator in each crosssection $z$ :

$$
\ddot{q}(z, t)+\Gamma_{\mathrm{m}} \dot{q}(z, t)+\Omega_{\mathrm{m}}^{2} q(z, t)=\frac{F(z, t)}{m_{\mathrm{eff}}}
$$

with $\frac{\Gamma_{\mathrm{m}}}{2 \pi}$ the Brillouin linewidth, $\Omega_{\mathrm{m}}^{2}=\frac{k_{\text {eff }}}{m_{\mathrm{eff}}}$ the angular frequency, $m_{\text {eff }}$ the effective mass of the mechanical mode per unit length and $F(z, t)$ the total force acting on that mode per unit length. Since this equation does not explicitly depend on $z, q(z, t)$ directly inherits its positiondependency from $F(z, t)$. Note that any propagation of phonons along the waveguide is neglected in this step. Each cross-section oscillates independently, reminiscent of the molecular vibration in Raman scattering ${ }^{\mathrm{S} 1, \mathrm{~S} 3}$. This assumption is justified by the very low group velocity of the acoustic phonons. From phase-matching, the acoustic wavevector along the $z$-axis is $K=k_{0}-k_{-1}=\frac{\Omega}{v_{\mathrm{g}}}$. 
Therefore, the acoustic phase velocity $v_{\mathrm{a}}=\frac{\Omega}{K}$ equals the optical group velocity $v_{\mathrm{g}}$. At the same time, we must have $v_{\mathrm{a}} v_{\mathrm{a}, \mathrm{g}}=c_{\mathrm{a}}^{2}$ with $v_{\mathrm{a}, \mathrm{g}}$ the acoustic group velocity and $c_{\mathrm{a}}$ the bulk acoustic velocity. Here we treat the silicon wire acoustically as a slab waveguide close to cut-off. This yields a low acoustic group velocity of $v_{\mathrm{a}, \mathrm{g}} \approx 1 \mathrm{~m} / \mathrm{s}$ for our silicon wires, which is confirmed by the finite-element model. Therefore we treat the acoustic wave as a localized oscillator, following the success of this description in other systems ${ }^{\mathrm{S} 1, \mathrm{~S} 2}$.

From power-conservation ${ }^{\mathrm{S} 4}$, the optical force $F(z, t)$ per unit length can be related to $\frac{\partial n_{\text {eff }}}{\partial q}$ as

$$
F(z, t)=\left.\frac{1}{c} \frac{\partial n_{\mathrm{eff}}}{\partial q}\right|_{q_{\mathrm{avg}}} P(z, t)
$$

The power $P(z, t)=2 E^{2}(z, t)$ contains frequencies $n \Omega \forall n$ up to the total number of lines. However, we assume that only the component at $\Omega$ excites the mechanical motion. So we take $F(z, t)=\frac{1}{2} f_{\Omega} \exp (i(\Omega t-K z))+$ c.c. with $f_{\Omega}=\frac{1}{c} \frac{\partial n_{\text {eff }}}{\partial q} p_{\Omega}$ and $p_{\Omega}=2 \sum_{n} a_{n} a_{n-1}^{\star}$.

We normalized the amplitudes $a_{n}$ such that the power of wave $\omega_{n}$ is $\left|a_{n}\right|^{2}$. Close to resonance $\left(\Omega \approx \Omega_{\mathrm{m}}\right.$ ), the steady-state response of the harmonic oscillator is $q_{\Omega}=Q_{\mathrm{m}} \frac{f_{\Omega}}{k_{\text {eff }}} \mathcal{L}(\Omega)$ with the Lorentzian function $\mathcal{L}(\Omega)=$ $\frac{1}{-2 \Delta_{r}+i}$, the relative detuning $\Delta_{r}=\frac{\Omega-\Omega_{\mathrm{m}}}{\Gamma_{\mathrm{m}}}$ and the quality factor $Q_{\mathrm{m}}=\frac{\Omega_{\mathrm{m}}}{\Gamma_{\mathrm{m}}}$. Therefore, we can write the nonlinear index change in terms of the nonlinear Kerr and Brillouin parameters $\gamma_{\mathrm{K}}$ and $\gamma_{\mathrm{SBS}}$ :

$$
\begin{aligned}
\Delta n_{\Omega} & =\Delta n_{\Omega, \text { Kerr }}+\Delta n_{\Omega, \text { Brillouin }} \\
& =\frac{\gamma_{\mathrm{K}}}{k_{0}} p_{\Omega}+\frac{\gamma_{\mathrm{SBS}}}{k_{0}} p_{\Omega} \mathcal{L}(\Omega) \\
& =\frac{p_{\Omega}}{k_{0}} \gamma(\Omega)
\end{aligned}
$$

where we defined the total nonlinearity parameter $\gamma(\Omega)=\gamma_{\mathrm{K}}+\gamma_{\mathrm{SBS}} \mathcal{L}(\Omega)$, using $\gamma_{\mathrm{K}} \equiv k_{0} \frac{\bar{n}_{2}}{A_{\text {eff }}}$ and $\gamma_{\mathrm{SBS}} \equiv$ $\omega_{0} \frac{Q_{\mathrm{m}}}{k_{\text {eff }}}\left(\frac{1}{c} \frac{\partial n_{\text {eff }}}{\partial q}\right)^{2}$. We note that this formula for the Brillouin nonlinearity is identical to the rigorous ${ }^{\mathrm{S} 5, \mathrm{~S} 6} \gamma_{\mathrm{SBS}}=$ $\omega_{0} Q_{\mathrm{m}}|\langle\mathbf{f}, \mathbf{u}\rangle|^{2} /\left(4 k_{\text {eff }}\right)$ if we identify $\frac{1}{c}\left|\frac{\partial n_{\text {eff }}}{\partial q}\right| \equiv \frac{|\langle\mathbf{f}, \mathbf{u}\rangle|}{2}$. Hence the evolution of the amplitudes is

$$
\begin{aligned}
\frac{\mathrm{d} a_{n}}{\mathrm{~d} z} & =-i \frac{k_{0}}{2}\left(\Delta n_{\Omega} a_{n-1}+\Delta n_{\Omega}^{\star} a_{n+1}\right) \\
\Delta n_{\Omega} & =\frac{p_{\Omega}}{k_{0}} \gamma(\Omega)=\frac{2 \gamma(\Omega)}{k_{0}} \sum_{n} a_{n} a_{n-1}^{\star}
\end{aligned}
$$

These equations can be solved analytically since $\Delta n_{\Omega}$ turns out to be a constant of motion. Indeed, derivation yields

$$
\begin{aligned}
\frac{\mathrm{d} \Delta n_{\Omega}}{\mathrm{d} z} & \propto \sum_{n}\left(a_{n} \frac{\mathrm{d} a_{n-1}^{\star}}{\mathrm{d} z}+\frac{\mathrm{d} a_{n}}{\mathrm{~d} z} a_{n-1}^{\star}\right) \\
& \propto \sum_{n} \Delta n_{\Omega}\left(\left|a_{n}\right|^{2}-\left|a_{n-1}\right|^{2}\right) \\
& +\Delta n_{\Omega}^{\star}\left(a_{n} a_{n-2}^{\star}-a_{n+1} a_{n-1}^{\star}\right) \\
& =0
\end{aligned}
$$

Consequently, equation (S1) can be solved either directly by using properties of the Bessel functions or indirectly by noting that $\Delta n_{\Omega}(z)=\Delta n_{\Omega}(0)$ such that the nonlinear interaction is equivalent to spatiotemporal phasemodulation. Specifically,

$$
\begin{aligned}
E(z, t)= & \frac{1}{2} \sum_{n} a_{n}(z) \exp \left(i\left(\omega_{n} t-k_{n} z\right)\right)+\text { c.c. } \\
= & \frac{1}{2} \exp \left(-i k_{0} z \Delta n_{\mathrm{eff}}(z, t)\right) \times \\
& \sum_{n} a_{n}(0) \exp \left(i\left(\omega_{n} t-k_{n} z\right)\right)+\text { c.c. }
\end{aligned}
$$

Moreover, we have

$$
\begin{aligned}
\Delta n_{\mathrm{eff}}(z, t) & =\left|\Delta n_{\Omega}(0)\right| \sin \left(\Omega t-K z+\varphi_{0}\right) \\
& =\frac{2|\gamma(\Omega)|}{k_{0}}\left|\sum_{n} a_{n}(0) a_{n-1}^{\star}(0)\right| \sin \left(\Omega t-K z+\varphi_{0}\right)
\end{aligned}
$$

with $\varphi_{0}=\angle\left\{\Delta n_{\Omega}(0) \exp \left(i \frac{\pi}{2}\right)\right\}$. As previously noted in the context of photonic crystal fibres ${ }^{\mathrm{S} 1}$, this is equivalent to phase-modulation with a depth $\xi$ determined by the strength of the input fields, the interaction length and the nonlinear parameter $|\gamma(\Omega)|$. The amplitudes of the individual components can finally be found by inserting $\exp (i \xi \sin \Phi)=\sum_{n} \mathcal{J}_{n}(\xi) \exp (i n \Phi)$ with $\mathcal{J}_{n}$ the $n$ th-order Bessel function of the first kind. To arrive at this phase-modulation picture, we assumed that all index changes originate from the beating at frequency $\Omega$. This is correct for the mechanical effect since it is weak off resonance. However, the Kerr response is nonresonant at telecom wavelengths. Thus its strength is the same at $\omega_{0}+n \Omega$ for all $n$. We include the $n \Omega(n \neq 1)$ Kerr-mediated coupling in the next paragraph, keeping in mind that equations (S2)-(S3) are only entirely correct when $\gamma_{\mathrm{K}}=0$.

To see how the modulation picture (S2)-(S3) relates to the traditional view of SBS as a pure gain process, we simplify equation (S1) to the case of an undepleted pump, a Stokes and an anti-Stokes signal. Neglecting higher-order cascading, this yields

$$
\begin{aligned}
& \frac{\mathrm{d} a_{\mathrm{s}}}{\mathrm{d} z}=-i \gamma^{\star}(\Omega)\left(\left|a_{\mathrm{p}}\right|^{2} a_{\mathrm{s}}+a_{\mathrm{p}}^{2} a_{\mathrm{a}}^{\star}\right) \\
& \frac{\mathrm{d} a_{\mathrm{a}}}{\mathrm{d} z}=-i \gamma(\Omega)\left(\left|a_{\mathrm{p}}\right|^{2} a_{\mathrm{a}}+a_{\mathrm{p}}^{2} a_{\mathrm{s}}^{\star}\right)
\end{aligned}
$$


In case $a_{\mathrm{a}}(0)=0$, the initial evolution of the Stokes power is

$$
\frac{\mathrm{d} P_{\mathrm{s}}}{\mathrm{d} z}=-2 \Im\{\gamma(\Omega)\} P_{\mathrm{p}} P_{\mathrm{s}}
$$

Since $\Im\{\gamma(\Omega)\}=-\frac{\gamma_{\mathrm{SBS}}}{4 \Delta_{r}^{2}+1}$, we recover a Lorentzian Brillouin gain profile in this approximation:

$$
\begin{aligned}
\frac{\mathrm{d} P_{\mathrm{s}}}{\mathrm{d} z} & =G_{\mathrm{SBS}}(\Omega) P_{\mathrm{p}} P_{\mathrm{s}} \\
G_{\mathrm{SBS}}(\Omega) & =\frac{2 \gamma_{\mathrm{SBS}}}{4 \Delta_{r}^{2}+1}
\end{aligned}
$$

Similarly, the anti-Stokes experiences a Lorentzian loss profile if $a_{\mathrm{s}}(0)=0$. Thus the Kerr effect has no impact on the initial evolution of the Stokes wave. Therefore, forward SBS is a pure gain process as long as the antiStokes build-up is negligible. By numerically integrating equations (S4), including linear losses, we confirm that this is the case in our experiments. The $n \Omega(n \neq 1)$ Kerr-mediated coupling does not change this conclusion. We can see this as follows. In the Lorentz-model for the permittivity, the Kerr response can be treated as a second-order nonlinear spring ${ }^{\mathrm{S} 3}$

$$
\ddot{x}+\Gamma_{\mathrm{e}} \dot{x}+\Omega_{e}^{2}(x) x=-\frac{e}{m_{\mathrm{e}}} E
$$

with $x$ the displacement of the electron cloud, $m_{\mathrm{e}}$ the electron mass, $\Omega_{\mathrm{e}}^{2}(x)=\frac{k_{\mathrm{e}}(x)}{m_{\mathrm{e}}}$ and $k_{\mathrm{e}}(x)=k_{\mathrm{e}}(0)+\frac{\partial^{2} k_{\mathrm{e}}}{\partial x^{2}} x^{2}$ the nonlinear spring constant. Since $\omega_{n} \ll \Omega_{\mathrm{e}}$, the oscillator responds instantaneously to the Lorentz-force $-e E$ :

$$
\Omega_{e}^{2}(x) x=-\frac{e}{m_{\mathrm{e}}} E
$$

Thus the linear solution is $x_{\mathrm{L}}(z, t)=\frac{-e}{k_{\mathrm{e}}(0)} E(z, t)$. In the first Born approximation, the nonlinear displacement is

$$
x_{\mathrm{NL}}=-\frac{1}{k_{\mathrm{e}}(0)} \frac{\partial^{2} k_{\mathrm{e}}}{\partial x^{2}} x_{\mathrm{L}}^{3}
$$

And the nonlinear polarization is $P^{\mathrm{NL}}=\epsilon_{0} \chi^{\mathrm{NL}} E=$ $-N e x_{\mathrm{NL}}$ with $N$ the atomic number density. This implies that the nonlinear polarization is proportional to $E^{3}(z, t)$. Unlike in the Brillouin case, the Lorentz oscillator does not filter out $0 \Omega, 2 \Omega, 3 \Omega$, etc. terms. Selecting the right components of $P^{\mathrm{NL}}$, we find that equations (S4) are modified to

$$
\begin{aligned}
& \frac{\mathrm{d} a_{\mathrm{s}}}{\mathrm{d} z}=-i \gamma^{\star}(\Omega)\left(\left|a_{\mathrm{p}}\right|^{2} a_{\mathrm{s}}+a_{\mathrm{p}}^{2} a_{\mathrm{a}}^{\star}\right)-i \gamma_{\mathrm{K}}\left|a_{\mathrm{p}}\right|^{2} a_{\mathrm{s}} \\
& \frac{\mathrm{d} a_{\mathrm{a}}}{\mathrm{d} z}=-i \gamma(\Omega)\left(\left|a_{\mathrm{p}}\right|^{2} a_{\mathrm{a}}+a_{\mathrm{p}}^{2} a_{\mathrm{s}}^{\star}\right)-i \gamma_{\mathrm{K}}\left|a_{\mathrm{p}}\right|^{2} a_{\mathrm{a}}
\end{aligned}
$$

for a strong, undepleted pump. The added terms on the right generate a constant phase shift and do, therefore, not alter the conclusion that these equations yield Brillouin gain when $a_{\mathrm{a}}(0)=0$. However, such added terms do invalidate the phase-modulation solution (S2)-(S3).

Back to that solution (S2)-(S3), at first sight we expect a Fano-like resonance for the Stokes power because the modulation depth depends on $|\gamma(\Omega)|$ and not on $\Im\{\gamma(\Omega)\}$. However, the input phase $\varphi_{0}$ also contains phase information on $\gamma(\Omega)$. We analytically check that the phase-modulation picture is equivalent to a pure gain process in the low-gain regime. Combining equations (S2) and (S3) with only an initial pump and Stokes wave, we find

$$
a_{\mathrm{s}}(z)=a_{\mathrm{s}}(0)-\mathcal{J}_{1}(\xi) a_{\mathrm{p}}(0) \exp \left(-i\left(\varphi_{0}+\pi\right)\right)
$$

with $\xi=2|\gamma(\Omega)| \sqrt{P_{\mathrm{s}}(0) P_{\mathrm{p}}} z$ the unitless cascading parameter. The power of the Stokes wave then becomes

$$
P_{\mathrm{s}}(z)=P_{\mathrm{s}}(0)\left(1-2 \Im\{\gamma(\Omega)\} P_{\mathrm{p}} z\right)+\frac{\xi^{2}}{4} P_{\mathrm{p}}
$$

Here we approximated the Bessel function as $\mathcal{J}_{1}(\xi) \approx \frac{\xi}{2}$, which is valid in the low- $\xi$ regime. The last term, containing $\xi^{2}$, gives rise to a Fano resonance but is smaller than the other terms in this regime. Taking the derivative and letting $z \rightarrow 0$, we indeed recover the gain equation (S5). In our experiments we reach values of $\xi \approx 0.4$ in the longest waveguides and at maximum pump power. To conclude, we can safely neglect higher-order cascading and treat forward SBS as a pure gain process driven exclusively by the Brillouin nonlinearity. In the presence of linear optical losses, the modified evolution of the Stokes wave is

$$
\begin{gathered}
\frac{\mathrm{d} P_{\mathrm{s}}}{\mathrm{d} z}=\left(G_{\mathrm{SBS}}(\Omega) P_{\mathrm{p}} \exp (-\alpha z)-\alpha\right) P_{\mathrm{s}} \\
G_{\mathrm{SBS}}(\Omega)=\frac{2 \gamma_{\mathrm{SBS}}}{4 \Delta_{r}^{2}+1}
\end{gathered}
$$

with $\alpha$ the linear optical loss and $P_{\mathrm{p}}$ the input pump power. The analytical solution of this equation is

$$
P_{s}(L)=P_{s}(0) \exp \left(G_{\mathrm{SBS}}(\Omega) P_{\mathrm{p}} L_{\mathrm{eff}}-\alpha L\right)
$$

with $L_{\text {eff }}=\frac{1-\exp (-\alpha L)}{\alpha}$ the effective interaction length. In the case of nonlinear losses $\alpha\left(P_{\mathrm{p}}\right)$ the equations can be integrated numerically.

\section{THE BRILLOUIN GAIN COEFFICIENT}

The Brillouin gain coefficient $G_{\mathrm{SBS}}\left(\Omega_{\mathrm{m}}\right)=2 \gamma_{\mathrm{SBS}}$ at the mechanical resonance $\left(\Omega=\Omega_{\mathrm{m}}\right)$ is given by

$$
\begin{aligned}
G_{\mathrm{SBS}}\left(\Omega_{\mathrm{m}}\right) & =2 \omega_{0} \frac{Q_{\mathrm{m}}}{k_{\mathrm{eff}}}\left(\frac{1}{c} \frac{\partial n_{\mathrm{eff}}}{\partial q}\right)^{2} \\
& =\omega_{0} \frac{Q_{\mathrm{m}}}{2 k_{\mathrm{eff}}}|\langle\mathbf{f}, \mathbf{u}\rangle|^{2}
\end{aligned}
$$


with $\omega_{0}$ the optical angular frequency $[\mathrm{Hz}], Q_{\mathrm{m}}$ the mechanical quality factor [-], $k_{\text {eff }}$ the effective stiffness per unit length $\left[\mathrm{N} / \mathrm{m}^{2}\right], c$ the speed of light $[\mathrm{m} / \mathrm{s}], \frac{\partial n_{\text {eff }}}{\partial q}$ the derivative of the optical mode index with respect to mechanical motion $[1 / \mathrm{m}], \mathbf{f}=\mathbf{f}_{\mathrm{rp}}+\mathbf{f}_{\mathrm{es}}$ the power-normalized force density $\left[\mathrm{N} /\left(\mathrm{m}^{3} \mathrm{~W}\right)\right]$ and $\langle\mathbf{f}, \mathbf{u}\rangle=\int \mathbf{f}^{\star} \cdot \mathbf{u} d A$ the overlap integral between the optical forces and the mechanical mode $\mathbf{u}[-]$. Note that we chose the mechanical mode profile $\mathbf{u}$ to be dimensionless, so the mechanical coordinate $q$ is expressed in $[\mathrm{m}]$. Therefore the overlap integral $\langle\mathbf{f}, \mathbf{u}\rangle$ has dimension $\left[\mathrm{s} / \mathrm{m}^{2}\right]$, as does $\frac{1}{c} \frac{\partial n_{\text {eff }}}{\partial q}$. The effective stiffness is defined as $k_{\text {eff }} \equiv \Omega_{\mathrm{m}}^{2} m_{\text {eff }}$, where $m_{\mathrm{eff}} \equiv\langle\mathbf{u}, \rho \mathbf{u}\rangle$ is the effective mass per unit length $[\mathrm{kg} / \mathrm{m}]$ with $\rho$ the mass density. Typically, the elastic mode profile $\mathbf{u}$ is normalized such that $\max (|\mathbf{u}|)=1$. Then we have $m_{\mathrm{eff}} \approx m$ (and $m_{\mathrm{eff}} \leq m$ ) with $m$ the true mass of the waveguide per unit length. With these definitions, the gain coefficient indeed has dimensions $\left[\mathrm{W}^{-1} \mathrm{~m}^{-1}\right]$ :

$$
\begin{aligned}
{\left[G_{\mathrm{SBS}}\left(\Omega_{\mathrm{m}}\right)\right] } & =\left[\omega_{0}\right]\left[\frac{Q_{\mathrm{m}}}{k_{\mathrm{eff}}}\right][\langle\mathbf{f}, \mathbf{u}\rangle]^{2} \\
& =\frac{1}{\mathrm{~s}} \frac{\mathrm{m}^{2}}{\mathrm{~N}} \frac{\mathrm{s}^{2}}{\mathrm{~m}^{4}} \\
& =\frac{\mathrm{s}}{\mathrm{Nm}^{2}} \\
& =\frac{1}{\mathrm{Wm}}
\end{aligned}
$$

Furthermore, the Brillouin gain (S7) is identical to formulas presented in earlier theoretical work ${ }^{\mathrm{S} 5, \mathrm{~S} 6}$. Specifically, the gain coefficient (formula (10) of Qiu $(2013)^{\mathrm{S} 6}$ ) is

$$
G_{\mathrm{SBS}}\left(\Omega_{\mathrm{m}}\right)=\frac{2 \omega_{0} Q_{\mathrm{m}}}{\Omega_{\mathrm{m}}^{2} v_{\mathrm{gp}} v_{\mathrm{gs}}} \frac{|\langle\tilde{\mathbf{f}}, \mathbf{u}\rangle|^{2}}{\left\langle\mathbf{E}_{\mathrm{p}}, \epsilon \mathbf{E}_{\mathrm{p}}\right\rangle\left\langle\mathbf{E}_{\mathrm{s}}, \epsilon \mathbf{E}_{\mathrm{s}}\right\rangle\langle\mathbf{u}, \rho \mathbf{u}\rangle}
$$

with $v_{\mathrm{gp}}$ and $v_{\mathrm{gs}}$ the pump and Stokes optical group velocity, $\mathbf{E}_{\mathrm{p}}$ and $\mathbf{E}_{\mathrm{s}}$ the pump and Stokes electric field distribution, $\epsilon$ the dielectric permittivity and $\tilde{\mathbf{f}}$ the force distribution. The total power in a guided wave is $P=$ $\frac{v_{\mathrm{g}}}{2}\langle\mathbf{E}, \epsilon \mathbf{E}\rangle$, so we get

$$
G_{\mathrm{SBS}}\left(\Omega_{\mathrm{m}}\right)=\frac{\omega_{0} Q_{\mathrm{m}}}{2 \Omega_{\mathrm{m}}^{2}} \frac{|\langle\tilde{\mathbf{f}}, \mathbf{u}\rangle|^{2}}{P_{\mathrm{p}} P_{\mathrm{s}} m_{\mathrm{eff}}}
$$

where we used $m_{\text {eff }}=\langle\mathbf{u}, \rho \mathbf{u}\rangle$. Defining the powernormalized force distribution $\mathbf{f}$ as $\mathbf{f} \equiv \frac{\tilde{\mathbf{f}}}{\sqrt{P_{\mathrm{p}} P_{\mathrm{s}}}}$, we arrive at formula (S7):

$$
G_{\mathrm{SBS}}\left(\Omega_{\mathrm{m}}\right)=\omega_{0} \frac{Q_{\mathrm{m}}}{2 k_{\mathrm{eff}}}|\langle\mathbf{f}, \mathbf{u}\rangle|^{2}
$$

Our finite-element calculations of the SBS coefficient are based on this formula. This theory completely reproduces the conventional backward SBS coefficients in the limit of transverse waveguide dimensions much larger than the free-space wavelength ${ }^{\mathrm{S} 5, \mathrm{~S} 6}$. It predicts strongly enhanced photon-phonon coupling in sub-wavelength waveguides - as in our silicon nanowires.

\section{MODEL OF THE XPM EXPERIMENTS}

In the cross-phase modulation (XPM) experiments, we study the phase modulation imprinted on a probe wave by a strong intensity-modulated pump. The pump and its sidebands are located at frequencies $\omega_{0}, \omega_{1}=\omega_{0}+$ $\Omega$ and $\omega_{-1}=\omega_{0}-\Omega$. The probe has frequency $\omega_{\mathrm{pr}}$. The four-wave mixing interaction between these waves imprints sidebands $\omega_{\mathrm{pr}} \pm \Omega$ on the probe. We monitor the power $P_{\text {imprint }}$ in the $\omega_{\text {imprint }}=\omega_{\mathrm{pr}}+\Omega$ sideband at the end of the waveguide as a function of $\Omega$.

If there were only Brillouin coupling between the waves, the effective index would be modulated exclusively at frequency $\Omega$. However, the Kerr effect responds equally well to the beat notes $\Delta_{0}=\omega_{0}-\omega_{\mathrm{pr}}$ and $\Delta_{-1}=\omega_{-1}-\omega_{\mathrm{pr}}$. So there are four pathways to $\omega_{\text {imprint }}$ :

$$
\begin{aligned}
& \omega_{\text {imprint }}=\omega_{\mathrm{pr}}+\left(\omega_{1}-\omega_{0}\right) \\
& \omega_{\text {imprint }}=\omega_{\mathrm{pr}}+\left(\omega_{0}-\omega_{-1}\right) \\
& \omega_{\text {imprint }}=\omega_{1}-\Delta_{0} \\
& \omega_{\text {imprint }}=\omega_{0}-\Delta_{-1}
\end{aligned}
$$

Both the Kerr and the Brillouin effect take the first two, but only the Kerr effect takes the latter two pathways. Therefore the Kerr effect manifests itself with double strength in these experiments. Building on the formalism of supplementary section I, we calculate the imprinted sideband power $P_{\text {imprint }}$. The index modulation is

$$
\begin{aligned}
\Delta n_{\mathrm{eff}}(z, t) & =\left|\Delta n_{\Omega}\right| \sin \left(\Omega t-K z+\varphi_{\Omega}\right) \\
& +\left|\Delta n_{\Delta_{0}}\right| \sin \left(\Delta_{0} t-\left(k_{0}-k_{\mathrm{pr}}\right) z+\varphi_{\Delta_{0}}\right) \\
& +\left|\Delta n_{\Delta_{-1}}\right| \sin \left(\Delta_{-1} t-\left(k_{-1}-k_{\mathrm{pr}}\right) z+\varphi_{\Delta_{-1}}\right)
\end{aligned}
$$

with the following definitions

$$
\begin{aligned}
\Delta n_{\Omega} & =\frac{p_{\Omega}}{k_{0}}\left\{\gamma_{\mathrm{K}}+\gamma_{\mathrm{SBS}} \mathcal{L}(\Omega)\right\} \\
\Delta n_{\Delta_{0}} & =\frac{p_{\Delta_{0}}}{k_{0}} \gamma_{\mathrm{K}} \\
\Delta n_{\Delta_{-1}} & =\frac{p_{\Delta_{-1}}}{k_{0}} \gamma_{\mathrm{K}}
\end{aligned}
$$

As before, we denote the angles $\varphi_{\mathrm{j}}=\angle\left\{\Delta n_{\mathrm{j}} \exp \left(i \frac{\pi}{2}\right)\right\}$. We also define a modulation depth $\xi_{\mathrm{j}}=k_{0} z\left|\Delta n_{\mathrm{j}}\right|$ for each beat note. Next, we insert equation (S8) in equation (S2) and apply the Bessel expansion $\exp (i \xi \sin \Phi)=$ $\sum_{n} \mathcal{J}_{n}(\xi) \exp (i n \Phi)$ to each of the beat notes. This re- 

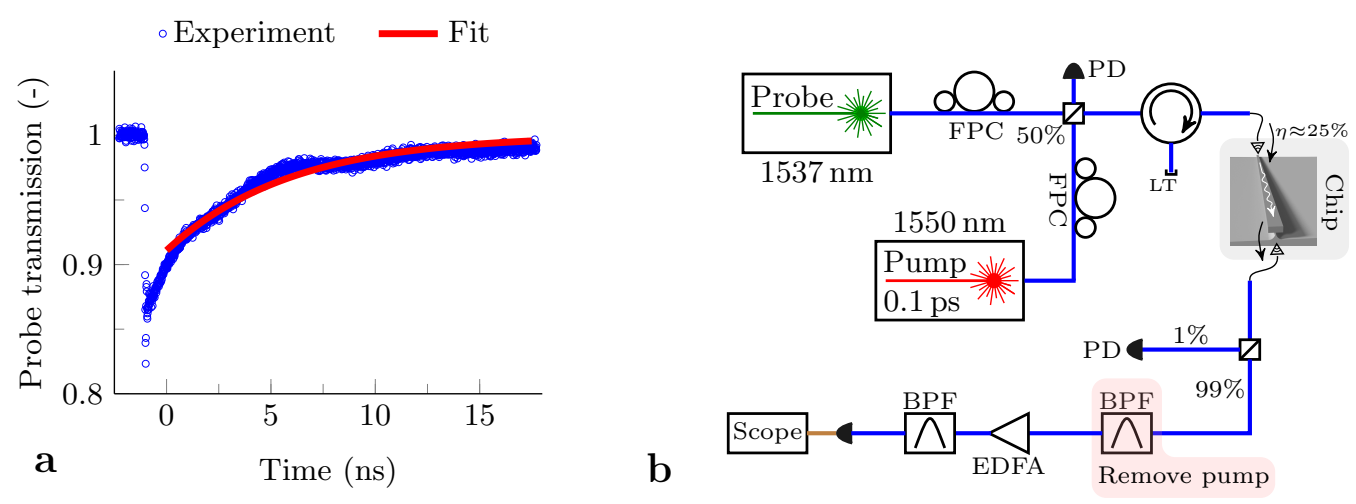

FIG. S1. Measurement of the free-carrier lifetime. a, Oscilloscope trace of the probe power. The pump pulse arrives at $t=-1 \mathrm{~ns}$. We start the fit a nanosecond later to avoid fitting to photodiode ringing artifacts. b, Pump-probe set-up used to obtain the trace. The band-pass filter (BPF) has more than $50 \mathrm{~dB}$ extinction at $1550 \mathrm{~nm}$.

sults in

$$
\begin{array}{r}
E(z, t)=\frac{1}{2} \sum_{k l m} \mathcal{J}_{k}\left(\xi_{\Omega}\right) \mathcal{J}_{l}\left(\xi_{\Delta_{0}}\right) \mathcal{J}_{m}\left(\xi_{\Delta_{-1}}\right) \times \\
\exp \left(-i\left(k \Phi_{\Omega}+l \Phi_{\Delta_{0}}+m \Phi_{\Delta_{-1}}\right)\right) \times \\
\sum_{n} a_{n}(0) \exp \left(i\left(\omega_{n} t-k_{n} z\right)\right)+\text { c.c. }
\end{array}
$$

Only three terms in the Bessel expansion influence $P_{\text {imprint }}$ when $\xi$ is small. In particular, for $(k l m)=$ $(-100),(010)$ and $(001)$ the frequencies $\omega_{\mathrm{pr}}, \omega_{1}$ and $\omega_{0}$ are shifted to $\omega_{\text {imprint }}$ respectively. Working out equation (S9) for these terms, we obtain

$$
\begin{aligned}
a_{\text {imprint }}(z)= & -\frac{\xi_{\Omega}}{2} \exp \left(i \varphi_{\Omega}\right) a_{\mathrm{pr}}+\frac{\xi_{\Delta_{0}}}{2} \exp \left(-i \varphi_{\Delta_{0}}\right) a_{1} \\
& +\frac{\xi_{\Delta_{-1}}}{2} \exp \left(-i \varphi_{\Delta_{-1}}\right) a_{0}
\end{aligned}
$$

for the amplitude $a_{\text {imprint }}(z)$ of the imprinted tone. Here we used $\mathcal{J}_{1}(\xi) \approx \frac{\xi}{2}$ for small $\xi$. Since the beat note amplitudes are $p_{\Omega}=2\left(a_{1} a_{0}^{\star}+a_{0} a_{-1}^{\star}\right), p_{\Delta_{0}}=2 a_{0} a_{\mathrm{pr}}^{\star}$ and $p_{\Delta_{-1}}=2 a_{-1} a_{\mathrm{pr}}^{\star}$, we finally obtain

$$
P_{\text {imprint }}(z)=\left|\gamma_{\mathrm{XPM}}(\Omega)\right|^{2}\left|p_{\Omega}\right|^{2} P_{\mathrm{pr}} \frac{z^{2}}{4}
$$

with $\gamma_{\mathrm{XPM}}(\Omega)=2 \gamma_{\mathrm{K}}+\gamma_{\mathrm{SBS}} \mathcal{L}(\Omega)$. Therefore we use the Fano lineshape $\left|\frac{\gamma_{\mathrm{XPM}}(\Omega)}{2 \gamma_{\mathrm{K}}}\right|^{2}$ as a fitting function for the normalized probe sideband power.

\section{MEASUREMENT OF THE FREE-CARRIER LIFETIME}

Free electrons and holes, created by two-photon absorption (TPA) in our experiments, induce significant free-carrier absorption (FCA) and free-carrier index changes (FCI) above a certain power threshold. As reflected in the saturation of the SBS gain (fig.3a), this threshold is about $25 \mathrm{~mW}$ in our $450 \times 230 \mathrm{~nm}$ silicon wires. From the observations

- that our finite-element and coupled-mode modelling of the Brillouin effect matches the experiments

- and that the off-resonance background is flat in the XPM experiment

we have evidence that the free carriers are not noticeably influencing our results below the threshold. Nevertheless, we performed a cross-FCA experiment (fig.S1) to exclude the possibility of a significant drop in free-carrier lifetime $\tau_{\mathrm{c}}$ caused by the underetch of our wires.

The pump was a $\approx 100$ fs-pulse with a repetition rate of $\frac{1}{50 \mathrm{~ns}}$ and peak power of $\approx 1 \mathrm{~kW}$. When a pump pulse arrives, it creates many free-carriers by TPA. The freecarriers recombine before the next pump pulse arrives. Their presence is read out by monitoring the power of a c.w. probe wave on a high-speed oscilloscope. Thus the transmission $T$ of the probe is

$$
T=\exp \left(-\alpha_{\mathrm{FCA}}(t)\right)=\exp \left(-\alpha_{\mathrm{FCA}}\left(t_{0}\right) \exp \left(-\frac{t}{\tau_{\mathrm{c}}}\right)\right)
$$

where we normalized the transmission to the case without FCA. Here we exploited the relation $\alpha_{\mathrm{FCA}}(t) \propto N(t)$ with $N(t)$ the free-carrier concentration.

The experiments yield typical values of $\tau_{\mathrm{c}}=6.2 \mathrm{~ns}$ before the etch and $\tau_{\mathrm{c}}=5.7 \mathrm{~ns}$ after the etch in identical waveguides. Hence there is, if at all, only a minor decrease of $\tau_{\mathrm{c}}$ due to the underetch. The associated bandwidth of $f_{3 \mathrm{~dB}}=\frac{1}{2 \pi \tau_{\mathrm{c}}}=28 \mathrm{MHz}$ suggests a negligible FCI-effect at $10 \mathrm{GHz}$. As a precaution, we work when possible - in the longer wires - with low power (below $15 \mathrm{~mW}$ on-chip) in the XPM-experiments. The free-carrier nonlinearity $\gamma_{\mathrm{FCI}}$ can, in principle, always be reduced below $\gamma_{\mathrm{K}}$ because $\gamma_{\mathrm{FCI}} \propto P_{\mathrm{pump}}$ while $\gamma_{\mathrm{K}}$ does not depend on $P_{\text {pump }}$. 


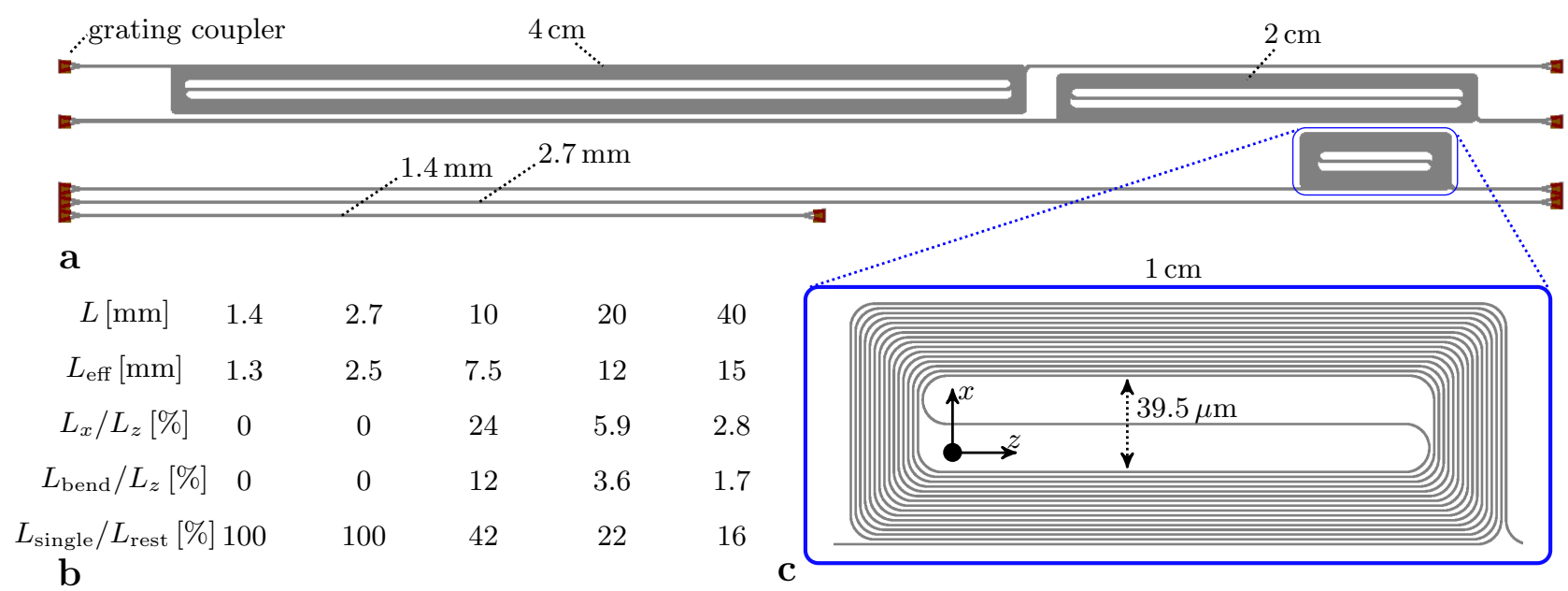

FIG. S2. Overview of the straight and spiral waveguides. a, Top view of the silicon wires on a pillar. The $1.4 \mathrm{~mm}-$ and $2.7 \mathrm{~mm}$-long wires are straight, the other waveguides are coiled up into a spiral. $\mathbf{b}$, Properties of the waveguides: $L$ is the total waveguide length and $L_{\text {eff }}=\frac{1-\exp (-\alpha L)}{\alpha}$ the effective length with $\alpha=2.6 \mathrm{~dB} / \mathrm{cm}$. The total wire length can be decomposed as $L=L_{x}+L_{z}+L_{\text {bend }}$ into the length $L_{x}$ along the $x$-axis, the length $L_{z}$ along the $z$-axis and the length of the bends $L_{\mathrm{bend}}$. The fractions $L_{x} / L_{z}$ and $L_{\text {bend }} / L_{z}$ drop rapidly as the spirals become longer. We also define the ratio $L_{\text {single }} / L_{\text {rest }}$ between the effective length $L_{\text {single }}$ of isolated wires (i.e. more than $10 \mu \mathrm{m}$ from another wire) and the effective length $L_{\text {rest }}$ of the wires in close proximity $(<2 \mu \mathrm{m})$. c, The $1 \mathrm{~cm}$-long spiral has the highest fraction of wires along the $x$-axis, the highest fraction of bends and the smallest fraction of wires in proximity of another wire. The spacing between adjacent wires is $1.55 \mu \mathrm{m}$.

\section{INHOMOGENEOUS BROADENING}

The sensitivity of the resonance frequency to width changes is $19.2 \mathrm{MHz} / \mathrm{nm}$ (fig.3b). Therefore, a width change of $2 \mathrm{~nm}$ shifts the resonance by as much as its $35 \mathrm{MHz}$ linewidth. The phononic resonance is more than an order of magnitude less sensitive to height variations $(2.3 \mathrm{MHz} / \mathrm{nm})$ and pillar size variations $(0.5 \mathrm{MHz} / \mathrm{nm})$. Hence geometrical non-uniformities, particularly in the wire width, may broaden the mechanical resonance. This potential broadening cannot account for most of the mechanical linewidth. Otherwise, the measured $Q_{\mathrm{m}}$-factors would not agree - within the measurement error - with the simulated leakage-limited $Q_{\mathrm{m}}$-factors (fig.4b). Thus other sources of acoustic linewidth must be small relative to the clamping loss.

Nevertheless, we estimate an upper limit on the broadening caused by width non-uniformities. These width fluctuations can be measured indirectly from variations in the optical mode index, and thus the center wavelength of ring resonators, Mach-Zehnder interferometers and arrayed waveguide gratings. The standard deviation of the long-range variations in center wavelength of such devices is less than $0.6 \mathrm{~nm}$ for our deep UV process ${ }^{\mathrm{S} 7}$. In our silicon wires, a $1 \mathrm{~nm}$ shift in waveguide width (height) yields a $1 \mathrm{~nm}(2 \mathrm{~nm})$ shift in center wavelength ${ }^{\mathrm{S} 7}$. Thus, width (height) variations typically $(1 \sigma)$ do not exceed $0.6 \mathrm{~nm}(0.3 \mathrm{~nm})$. Therefore, the width (height) fluctuations contribute less than $11 \mathrm{MHz}(0.7 \mathrm{MHz})$ or $31 \%$ $(2 \%)$ of the measured linewidth. Besides, it was recently shown that $300 \mathrm{~mm}$-diameter (instead of $200 \mathrm{~mm}$ diameter) wafer processing results in a factor 2 better width and height uniformity ${ }^{\mathrm{S} 8}$.

Furthermore, the pillar size broadening is smaller than the width-induced broadening. By repeated pillar size measurements (as in fig.1c) in different sections of the spirals, we find that the pillar size variation falls within the $\pm 5 \mathrm{~nm}$ error of the scanning electron microscope. This yields a linewidth contribution of less than $4 \mathrm{MHz}$.

In conclusion, there is no evidence for inhomogeneous broadening caused by geometrical non-uniformities. The measured and simulated leakage-limited $Q_{\mathrm{m}}$-factors are - within the measurement error - in good agreement (fig.4b). The upper limits on width-, height- and pillarinduced broadening are $11 \mathrm{MHz}, 0.7 \mathrm{MHz}$ and $4 \mathrm{MHz}$.

\section{PEAK SPLITTING IN SPIRALS}

As discussed in section $\mathrm{V}$, there is no evidence for inhomogeneous broadening of the resonances - in the sense that a geometric parameter varies continuously along the wire. However, our experiments do show peak splitting in the $1 \mathrm{~cm}$ spiral (fig.S2c). This splitting occurs in both the XPM (fig.S3) and gain experiment (fig.S4).

We find that the Fano resonances (fig.S3) are nearly identical in the $2.7 \mathrm{~mm}$ straight wire and the $2 \mathrm{~cm}$ and $4 \mathrm{~cm}$ spirals. Thus neither the interaction strength $\gamma_{\mathrm{SBS}}$ nor the linewidth $\frac{\Gamma_{m}}{2 \pi}$ is affected by the wire length. The Fano resonance shows two sharp peaks in the $1 \mathrm{~cm}$ spiral (fig.S3b). The peaks are separated by $55 \mathrm{MHz}$. This particular spiral also has a reduced $\gamma_{\mathrm{SBS}}$.

Similarly, the gain coefficient $2 \gamma_{\text {SBS }}$ and the linewidth $\frac{\Gamma_{\mathrm{m}}}{2 \pi}$ are nearly identical in the $2.7 \mathrm{~mm}$ straight wire and 


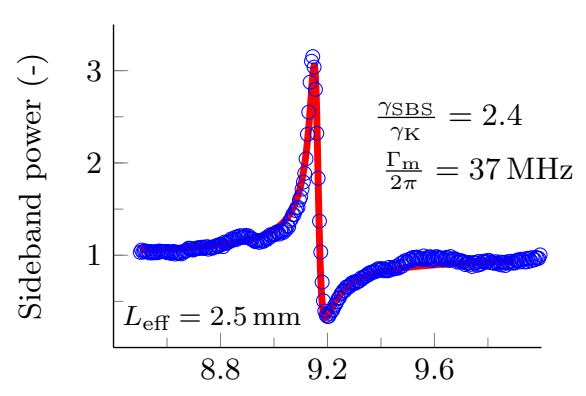

a Frequency spacing $\frac{\Omega}{2 \pi}(\mathrm{GHz})$

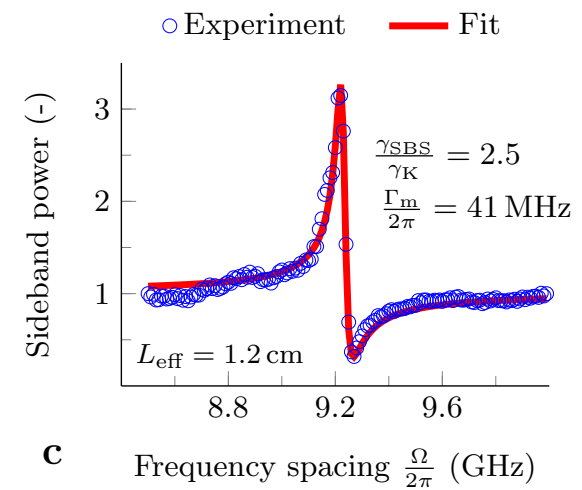

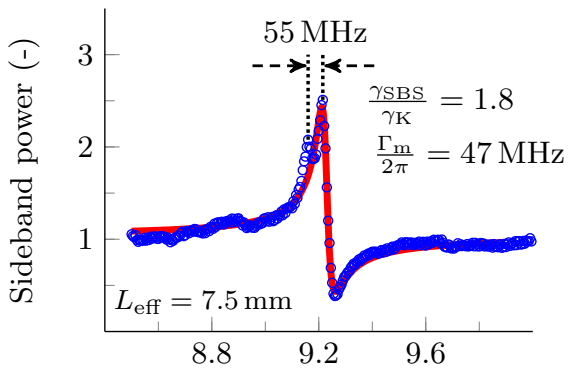

b Frequency spacing $\frac{\Omega}{2 \pi}(\mathrm{GHz})$

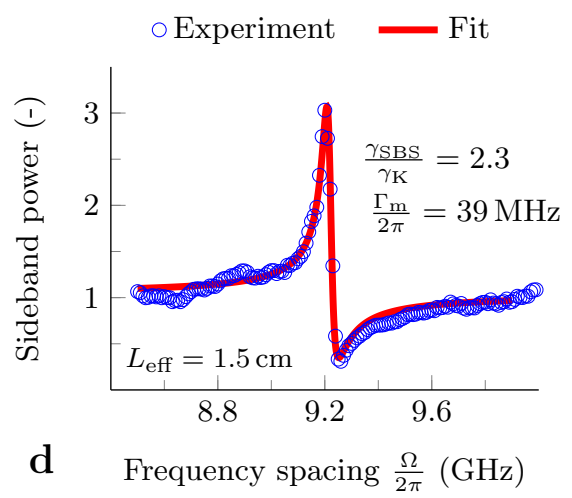

FIG. S3. Fano resonances in wires of increasing length. We conduct the XPM-experiment (fig.2d) in wires of lengths $1.4 \mathrm{~mm}$ (not shown), $2.7 \mathrm{~mm}(\mathbf{a}), 1 \mathrm{~cm}(\mathbf{b}), 2 \mathrm{~cm}(\mathbf{c})$ and $4 \mathrm{~cm}(\mathbf{d})$. The resonance is identical in the $1.4 \mathrm{~mm}$ and the $2.7 \mathrm{~mm}$ wires. The extracted Brillouin nonlinearity $\gamma_{\mathrm{SBS}}$ and linewidth $\frac{\Gamma_{\mathrm{m}}}{2 \pi}$ are nearly identical in the $2.7 \mathrm{~mm}$ straight wire, the $2 \mathrm{~cm}$ spiral and the $4 \mathrm{~cm}$ spiral. Only the $1 \mathrm{~cm}$ spiral (b) exhibits peak splitting and a reduced $\gamma_{\mathrm{SBS}}$. The peak is split by $55 \mathrm{MHz}$.

the $2 \mathrm{~cm}$ and $4 \mathrm{~cm}$ spirals (fig.S4). The $1 \mathrm{~cm}$ spiral again consists of two resonances separated by $55 \mathrm{MHz}$ (fig.S4b). There is also a small remnant of the minor peak in the $2 \mathrm{~cm}$ and $4 \mathrm{~cm}$ spirals, which was not visible in the XPMexperiments because of the larger frequency step. Apart from the $1 \mathrm{~cm}$ spiral, the observed and predicted $\left(G_{\text {pred }}=\right.$ $\left.2 \gamma_{\mathrm{SBS}} P_{\mathrm{p}} L_{\text {eff }}\right)$ gain factors are in good agreement.

The $55 \mathrm{MHz}$ splitting may be explained by a $2.8 \mathrm{~nm}$ shift in wire width in a sub-section of the waveguide. We suspect that this is caused by the lithographical proximity effect, in which closely adjacent wires are slightly less wide than an isolated wire. Such proximity effects are known to play an important role in deep UV lithography ${ }^{\mathrm{S} 9}$. A drop in wire width would cause an upshift in resonance frequency, as we indeed observe going from the $2.7 \mathrm{~mm}$ straight wire to the spirals (fig.S3-S4).

For the relative height of the sub-peak and the main resonance (fig.S4b), we find $G_{\text {sub }} / G_{\text {main }}=45 \%$. This fraction corresponds to the ratio $L_{\text {single }} / L_{\text {rest }}=42 \%$ (fig.S2b), with $L_{\text {single }}$ the effective length of the waveguide where there is only a single wire (i.e. separated from other wires by more than $10 \mu \mathrm{m})$ and $L_{\text {rest }}$ the effective length of the spiral section where wires are in close proximity $(<2 \mu \mathrm{m})$. The spirals are not located in the middle between the gratings (fig.S2). Therefore, a detailed comparison also depends on the order of the wires.

In conclusion, the $1 \mathrm{~cm}$ spiral shows a reduced Bril- louin nonlinearity. We suspect that this is caused by the lithographical proximity effect, which reduces the width of closely adjacent wires. The $2 \mathrm{~cm}$ and $4 \mathrm{~cm}$ spiral exhibit a Brillouin nonlinearity at full strength; as strong as in the $2.7 \mathrm{~mm}$ straight wire (fig.S3-S4).

\section{MEASUREMENT OF BACKWARD SBS}

So far we focused on forward SBS, in which the excited phonons have a very short wavevector $K=\frac{\Omega}{v_{\mathrm{g}}}$ because the pump and Stokes have nearly equal wavevectors. However, the phononic mode demonstrated in this work (fig.1d) can also be operated at another point in its dispersion diagram. When the Stokes and pump counterpropagate through the wire, they generate the class of phonons that obey $K=2 k_{0}$. The propagating version (fig.S5a) of the Fabry-Pérot mechanical mode (fig.1d) may then induce gain as well. These modes have an acoustic group velocity that nearly equals the bulk acoustic velocity. Therefore, they have a significantly larger acoustic decay length $(\sim 10 \mu \mathrm{m})$ - making them more attractive for phononic circuits. We reconfigured our gain experiment (fig.S5b) to study such modes.

We also find a Lorentzian gain profile (fig.S5c-d), but this time at $13.66 \mathrm{GHz}$. For instance, we observe on/off gain of $0.22 \mathrm{~dB}$ with $P_{\mathrm{p}}=11.8 \mathrm{~mW}$ and $L=2 \mathrm{~cm}$ 

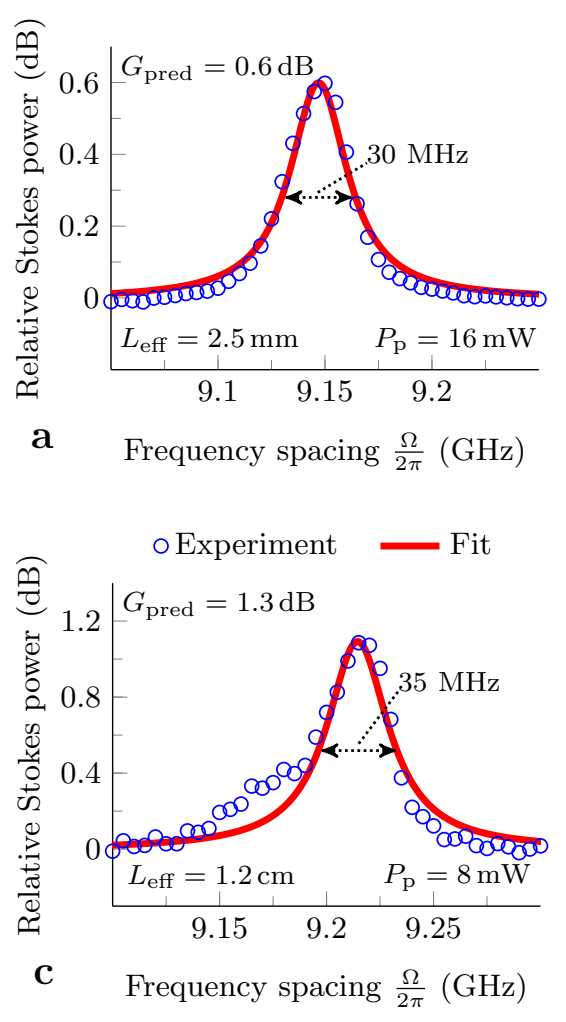
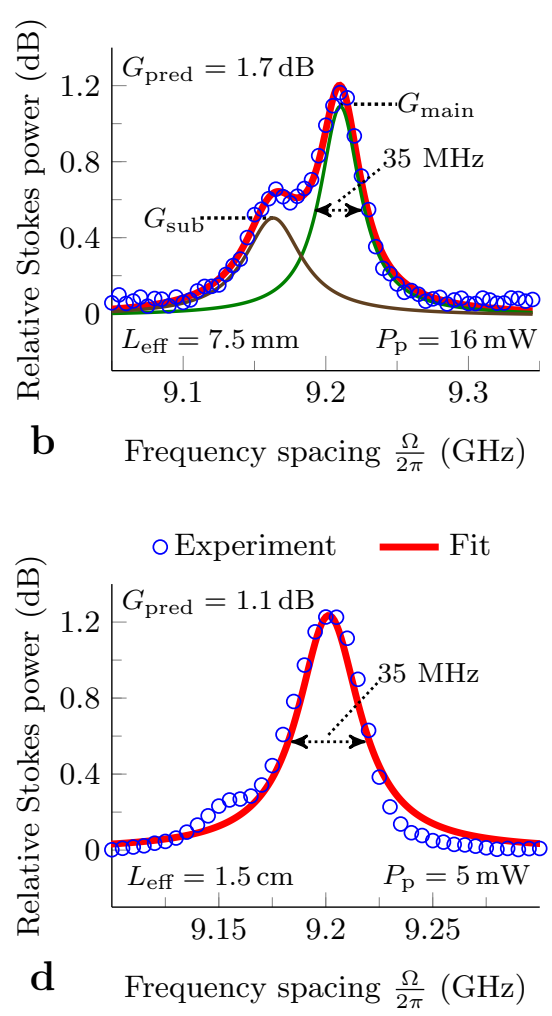

FIG. S4. Lorentzian gain resonances in wires of increasing length. We conduct the gain experiment (fig.2b) in wires of lengths $2.7 \mathrm{~mm}(\mathbf{a}), 1 \mathrm{~cm}(\mathbf{b}), 2 \mathrm{~cm}(\mathbf{c})$ and $4 \mathrm{~cm}(\mathbf{d})$. The $1 \mathrm{~cm}$ spiral shows two resonances: the minor peak (brown) and the main peak (green) add up to the entire resonance (red). We also show the predicted gain $G_{\text {pred }}=2 \gamma_{\mathrm{SBS}} P_{\mathrm{p}} L_{\mathrm{eff}}$ given $2 \gamma_{\mathrm{SBS}}=3218 \mathrm{~W}^{-1} \mathrm{~m}^{-1}$. The predicted and observed Brillouin gain match closely in the $2.7 \mathrm{~mm}$ straight wire, the $2 \mathrm{~cm}$ spiral and the $4 \mathrm{~cm}$ spiral. Only the $1 \mathrm{~cm}$ spiral (b) exhibits a reduced gain coefficient, caused by the peak splitting. Similar to the XPM-experiment, the peak is split by $55 \mathrm{MHz}$. There is a remnant of the minor peak in the $2 \mathrm{~cm}$ and $4 \mathrm{~cm}$ spirals. The linewidth is about $35 \mathrm{MHz}$ in all resonances, where we show the width of only the main resonance in the $1 \mathrm{~cm}$ spiral.

(fig.S5d). We extract $2 \gamma_{\mathrm{SBS}}=359 \mathrm{~W}^{-1} \mathrm{~m}^{-1}$ and $Q_{\mathrm{m}}=$ 971. Thus we have $\frac{2 \gamma_{\mathrm{SBS}}}{Q_{\mathrm{m}}}=0.37 \mathrm{~W}^{-1} \mathrm{~m}^{-1}$ : a factor 30 lower than in the forward case (fig.4c). We attribute this reduction to destructive interference between electrostriction and radiation pressure, as predicted before ${ }^{\mathrm{S} 6}$ for fully suspended wires. Because of this low overlap, we observe these resonances only in the long spirals (fig.S2). Based on our finite-element models, we expect this propagating mode (fig.S5a) at $14.4 \mathrm{GHz}$ with a coupling of $\frac{2 \gamma_{\mathrm{SBS}}}{Q_{\mathrm{m}}}=0.41 \mathrm{~W}^{-1} \mathrm{~m}^{-1}$. Therefore, we suspect that this is indeed the observed mode. Further investigations should resolve this issue, as the simulations predict that there are propagating modes with slightly better coupling at higher frequencies. However, even the highest simulated coupling strength reaches only $\frac{2 \gamma_{\mathrm{SBS}}}{Q_{\mathrm{m}}}=1.04 \mathrm{~W}^{-1} \mathrm{~m}^{-1}$ for an elastic mode at $27.3 \mathrm{GHz}$. The sweep of the frequency spacing $\frac{\Omega}{2 \pi}$ between the pump and Stokes seed was limited to $16 \mathrm{GHz}$ in the current set-up.

Finally, it has been predicted that backward SBS is suppressed in waveguides consisting of a cascade of fully suspended regions ${ }^{\mathrm{S} 10}$. The issue is that the acoustic wave cannot build up to its full strength in a suspended waveguide shorter than the acoustic decay length. In the limit of long suspended regions, the length of each region is effectively reduced by one acoustic decay length $(\sim 10 \mu \mathrm{m})$. However, our wires are partially suspended all along their length. Therefore the acoustic build-up has little effect on the backward SBS gain.
[S1] Kang, M., Nazarkin, A., Brenn, A. \& Russell, P. Tightly trapped acoustic phonons in photonic crystal fibres as highly nonlinear artificial Raman oscillators. Nature Phys. 5, 276-280 (2009).

[S2] Wang, J., Zhu, Y., Zhang, R. \& Gauthier, D. FSBS resonances observed in a standard highly nonlinear fiber.
Opt. Express 19, 5339-49 (2011).

[S3] Boyd, R. Nonlinear optics (Elsevier, London, 2008), 3 edn.

[S4] Rakich, P., Popović, M. \& Wang, Z. General treatment of optical forces and potentials in mechanically variable photonic systems. Opt. Express 17, 18116-35 (2009). 


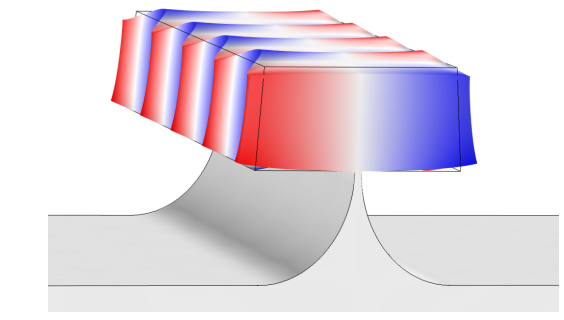

a

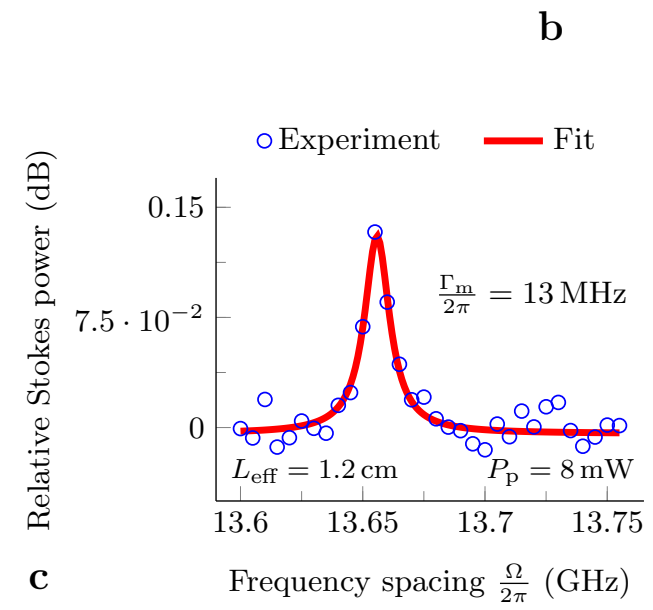

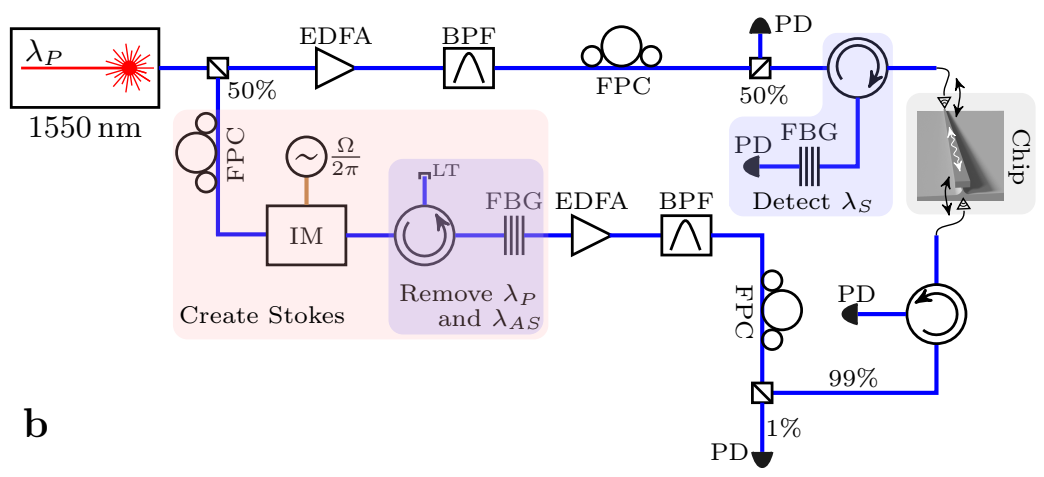

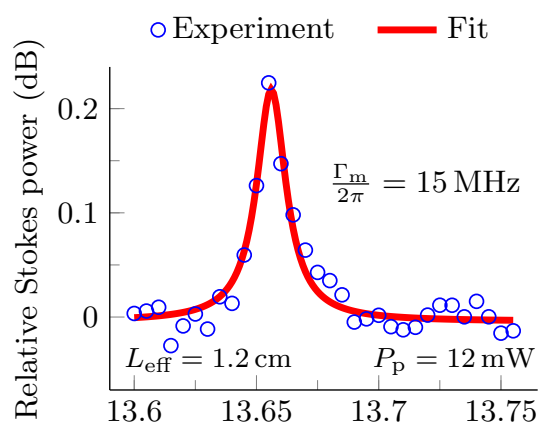

d Frequency spacing $\frac{\Omega}{2 \pi}(\mathrm{GHz})$

FIG. S5. Characterization of the backward Brillouin scattering. a, Propagating version of the Fabry-Pérot phononic mode (see fig.1d for comparison). b, Experimental set-up used to observe the backward SBS gain. This time the Stokes and pump wave counterpropagate through the chip, exciting phonons that satisfy $K=2 k_{0}$. These acoustic waves have a significantly longer decay length on the order of $10 \mu \mathrm{m}$. c-d, Lorentzian gain profile on the Stokes seed in the $2 \mathrm{~cm}$ spiral $\left(L_{\mathrm{eff}}=1.2 \mathrm{~cm}\right)$ for on-chip pump powers $P_{\mathrm{p}}$ of $8 \mathrm{~mW}(\mathbf{c})$ and $12 \mathrm{~mW}(\mathbf{d})$.

[S5] Rakich, P., Reinke, C., Camacho, R., Davids, P. \& Wang, Z. Giant enhancement of stimulated Brillouin scattering in the subwavelength limit. Phys. Rev. X 2, 1-15 (2012).

[S6] Qiu, W. et al. Stimulated Brillouin scattering in nanoscale silicon step-index waveguides: a general framework of selection rules and calculating SBS gain. Opt. Express 21, 31402 (2013).

[S7] Selvaraja, S., Bogaerts, W., Dumon, P., Van Thourhout, D. \& Baets, R. Subnanometer linewidth uniformity in silicon nanophotonic waveguide devices using CMOS fabrication technology. IEEE J. Sel. Top.
Quantum Electron. 1-9 (2010).

[S8] Selvaraja, S. et al. Highly uniform and low-loss passive silicon photonics devices using a $300 \mathrm{~mm}$ CMOS platform. OFC 3-5 (2014).

[S9] Bogaerts, W. et al. Nanophotonic waveguides in siliconon-insulator fabricated with CMOS technology. J. Lightwave Technol. 23, 401-412 (2005).

[S10] Wolff, C., Steel, M., Eggleton, B. \& Poulton, C. Stimulated Brillouin Scattering in integrated photonic waveguides: forces, scattering mechanisms and coupled mode analysis. arXiv preprint 1-16 (2014). 